\title{
SPHERICAL HARMONIC ANALYSIS ON AFFINE BUILDINGS
}

\author{
JAMES PARKINSON \\ School of Mathematics and Statistics \\ University of Sydney, NSW 2006 \\ Australia \\ jamesp@maths.usyd.edu.au
}

October 16,2018

\begin{abstract}
Let $\mathscr{X}$ be a locally finite regular affine building with root system $R$. There is a commutative algebra $\mathscr{A}$ spanned by averaging operators $A_{\lambda}$, $\lambda \in P^{+}$, acting on the space of all functions $f: V_{P} \rightarrow \mathbb{C}$, where $V_{P}$ is in most cases the set of all special vertices of $\mathscr{X}$, and $P^{+}$is a set of dominant coweights of $R$. This algebra is studied in [6] and 7 for $\tilde{A}_{n}$ buildings, and the general case is treated in 15 .

In this paper we show that all algebra homomorphisms $h: \mathscr{A} \rightarrow \mathbb{C}$ may be expressed in terms of the Macdonald spherical functions. We also provide a second formula for these homomorphisms in terms of an integral over the boundary of $\mathscr{X}$. We may regard $\mathscr{A}$ as a subalgebra of the $C^{*}$-algebra of bounded linear operators on $\ell^{2}\left(V_{P}\right)$, and we write $\mathscr{A}_{2}$ for the closure of $\mathscr{A}$ in this algebra. We study the Gelfand map $\mathscr{A}_{2} \rightarrow \mathscr{C}\left(M_{2}\right)$, where $M_{2}=$ $\operatorname{Hom}\left(\mathscr{A}_{2}, \mathbb{C}\right)$, and we compute $M_{2}$ and the Plancherel measure of $\mathscr{A}_{2}$. We also compute the $\ell^{2}$-operator norms of the operators $A_{\lambda}, \lambda \in P^{+}$, in terms of the Macdonald spherical functions.
\end{abstract}

\section{INTRODUCTION}

In 15] we showed that under the weak hypothesis of regularity, to each affine building $\mathscr{X}$ of irreducible type there is naturally associated a commutative algebra $\mathscr{A}$ spanned by vertex set averaging operators $A_{\lambda}, \lambda \in P^{+}$, acting on the space of all functions $f: V_{P} \rightarrow \mathbb{C}$. Here $P^{+}$is the set of dominant coweights of an appropriate root system $R$, and $V_{P}$ is in most cases the set of all special vertices of $\mathscr{X}$.

Let $\mathbb{C}[P]$ denote the group algebra of the coweight lattice $P$ of $R$, with the group operation written multiplicatively. Thus elements of $\mathbb{C}[P]$ are linear combinations of the formal exponentials $\left\{x^{\lambda}\right\}_{\lambda \in P}$, and $x^{\lambda} x^{\mu}=x^{\lambda+\mu}=x^{\mu} x^{\lambda}$ for all $\lambda, \mu \in P$. Let $W_{0}$ denote the Weyl group of $R$. Thus $W_{0}$ acts on $P$, and hence on $\mathbb{C}[P]$ by linearly extending $w x^{\lambda}=x^{w \lambda}$. Let $\mathbb{C}[P]^{W_{0}}=\left\{f \in \mathbb{C}[P] \mid w f=f\right.$ for all $\left.w \in W_{0}\right\}$. In [15. Theorem 6.16] we showed that $\mathscr{A} \cong \mathbb{C}[P]^{W_{0}}$, with the isomorphism given by $A_{\lambda} \mapsto P_{\lambda}(x)$, where the elements $P_{\lambda}(x) \in \mathbb{C}[P]^{W_{0}}, \lambda \in P^{+}$, are the Macdonald spherical functions.

In this paper we study the algebra homomorphisms $h: \mathscr{A} \rightarrow \mathbb{C}$. The isomorphism $\mathscr{A} \cong \mathbb{C}[P]^{W_{0}}$ gives one formula for these homomorphisms in terms of the Macdonald spherical functions. The main part of this paper is devoted to a rather

2000 Mathematics Subject Classification. 20E42 (20C08 43A90).

Key words and phrases. Affine buildings, Macdonald spherical functions, harmonic functions. 
different line of reasoning which furnishes a second formula for the algebra homomorphisms $h: \mathscr{A} \rightarrow \mathbb{C}$ in terms of an integral over the boundary of $\mathscr{X}$ (that is, the spherical building at infinity). This formula is an analogue of the formula in 12. Proposition 3.3.1], which expresses the zonal spherical functions on a group $G$ of p-adic type as an integral over $K$, where $K$ is a certain compact subgroup of $G$. For example, $G=S L(n+1, F)$, where $F$ is a $p$-adic field, and $K=S L(n+1, \mathcal{O})$, where $\mathcal{O}$ is the ring of integers in $F$. See below for more details.

The algebra $\mathscr{A}$ may be regarded as a subalgebra of the $C^{*}$-algebra $\mathscr{L}\left(\ell^{2}\left(V_{P}\right)\right)$ of bounded linear operators on $\ell^{2}\left(V_{P}\right)$, and the closure $\mathscr{A}_{2}$ of $\mathscr{A}$ in $\mathscr{L}\left(\ell^{2}\left(V_{P}\right)\right)$ is a commutative $C^{*}$-algebra. We study the Gelfand map $\mathscr{A}_{2} \rightarrow \mathscr{C}\left(M_{2}\right)$, where $M_{2}=$ $\operatorname{Hom}\left(\mathscr{A}_{2}, \mathbb{C}\right)$. We compute $M_{2}$ and the Plancherel measure $\pi$ on $M_{2}$ corresponding to the natural state $A \mapsto\left(A \delta_{o}\right)(o)$ on $\mathscr{A}_{2}$ (here $o \in V_{P}$ is any fixed vertex, and $\delta_{o}(x)=1$ if $x=o$ and 0 otherwise). These results will be used both in this paper (for example, in the important Theorem 6.2 where we show the equality of our two expressions for the homomorphisms $h: \mathscr{A} \rightarrow \mathbb{C}$ ) and in later work, where we will study isotropic random walks on affine buildings. In Theorem 6.3 we compute the $\ell^{2}$-operator norms $\left\|A_{\lambda}\right\|, \lambda \in P^{+}$, in terms of the Macdonald spherical functions.

It is important to notice that throughout this work we do not assume the existence of any groups acting in particular ways on the building. We only use the building axioms, which puts our results into a general setting.

Let us briefly describe the connection between our work and that in 12. Let $G$ be a group of $p$-adic type with a maximal compact subgroup $K$, as in [12, §2.7]. Associated to $G$ there is an irreducible (but not necessarily reduced) root system $R$, as in [12 Chapter II]. As mentioned above, the typical example here is $G=S L(n+1, F)$ and $K=S L(n+1, \mathcal{O})$, where $F$ is a $p$-adic field and $\mathcal{O}$ is the ring of integers in $F$. In this case $R$ is a root system of type $A_{n}$.

A function $f: G \rightarrow \mathbb{C}$ is called bi-K-invariant if $f(g k)=f(k g)=f(g)$ for all $g \in G$ and $k \in K$. Let $\mathscr{L}(G, K)$ be the space of continuous, compactly supported bi- $K$-invariant functions on $G$. In [12 Theorem 3.3.6], Macdonald shows that $\mathscr{L}(G, K) \cong \mathbb{C}[Q]^{W_{0}}$ (the subalgebra of $W_{0}$-invariant elements of the group algebra of the coroot lattice $Q$ of $R$ ), and thus $\mathscr{L}(G, K)$ is a commutative convolution algebra.

A function $\phi: G \rightarrow \mathbb{C}$ is called a zonal spherical function relative to $K$ if

(i) $\phi(1)=1$,

(ii) $\phi$ is bi- $K$-invariant and continuous, and

(iii) $f * \phi=\lambda_{f} \phi$ for all $f \in \mathscr{L}(G, K)$, where $\lambda_{f}$ is a scalar

(see [12, Proposition 1.2.5]). In [12, Proposition 3.3.1] Macdonald gives a formula for the zonal spherical functions in terms of an integral over $K$, and in [12] Theorem 4.1.2] he uses this integral formula to obtain a second 'summation' formula for the spherical functions in terms of a sum over $W_{0}$ (the Weyl group of $R$ ) of rational functions.

The group $G$ acts strongly transitively on its Bruhat-Tits building $\mathscr{X}$ (see [12, Lemma 2.4.4]), which is locally finite, regular and affine, although Macdonald makes little use of this building. The contents of this paper (and [15]) lead us to the conclusion that, rather than playing a relatively minor role, the building theoretic elements alone determine the nature of the algebra $\mathscr{L}(G, K)$ and the zonal spherical functions. 
Specifically, the algebra $\mathscr{L}(G, K)$ is isomorphic to a subalgebra $\mathscr{A}_{0}$ of $\mathscr{A}$, spanned by the operators $\left\{A_{\lambda} \mid \lambda \in Q \cap P^{+}\right\}$. The reason that this smaller algebra occurs here is that Macdonald supposes that his groups of $p$-adic type are simply connected, and so for him the coweight lattice has to be replaced by the coroot lattice, and thus the set $V_{P}$ is replaced by the smaller set $V_{Q}$ consisting of all vertices of one special type. See [8, Propositions 2.4 and 2.5] for details in the $R=A_{2}$ case. In [15], Theorem 6.16] we showed that $\mathscr{A} \cong \mathbb{C}[P]^{W_{0}}$ (and indeed one can easily see that $\mathscr{A}_{0} \cong \mathbb{C}[Q]^{W_{0}}$ ), thus proving the analogue of [12, Theorem 3.3.6].

The zonal spherical functions on $G$ correspond to the spherical functions on $V_{Q}$ (see Definition [3.20), which in turn correspond to the algebra homomorphisms $h: \mathscr{A}_{0} \rightarrow \mathbb{C}$ (see Proposition 3.21). Our analogue of Macdonald's integral formula is Theorem 3.22 (see also Corollary 3.23), and our analogue of his summation formula is (2.1). Both of our formulae are proved in the context of the larger algebra $\mathscr{A}$ (in fact we will have little to say regarding the smaller algebra $\mathscr{A}_{0}$ in what follows). Since there are locally finite regular affine buildings that are not the Bruhat-Tits buildings of any group, our building approach puts the results of [12 into a more general setting.

Acknowledgements. The author would like to thank Donald Cartwright for many helpful suggestions throughout the preparation of this paper.

\section{Notations and Definitions}

1.1. Root Systems. Root systems play a significant role in this work. We fix the following notations and conventions, generally following [2] Chapter VI].

Let $R$ be an irreducible, but not necessarily reduced, root system in a real vector space $E$ with inner product $\langle\cdot, \cdot\rangle$. The rank of $R$ is $n$, the dimension of $E$. Let $I_{0}=\{1,2, \ldots, n\}$ and $I=\{0,1,2, \ldots, n\}$. Let $B=\left\{\alpha_{i} \mid i \in I_{0}\right\}$ be a fixed base of $R$, and write $R^{+}$for the set of positive roots (relative to $B$ ). For $\alpha \in E \backslash\{0\}$, write $\alpha^{\vee}=\frac{2 \alpha}{\langle\alpha, \alpha\rangle}$, and let $R^{\vee}=\left\{\alpha^{\vee} \mid \alpha \in R\right\}$ be the dual root system of $R$. Since $R$ is irreducible there is a unique highest root $\tilde{\alpha}$, and we define numbers $\left\{m_{i}\right\}_{i \in I}$ by $m_{0}=1$, and $\tilde{\alpha}=\sum_{i \in I_{0}} m_{i} \alpha_{i}$. For each $i \in I_{0}$ define $\lambda_{i} \in E$ by $\left\langle\lambda_{i}, \alpha_{j}\right\rangle=\delta_{i, j}$. The elements $\left\{\lambda_{i}\right\}_{i \in I_{0}}$ are called the fundamental coweights of $R$. The coweight lattice of $R$ is $P=\sum_{i \in I_{0}} \mathbb{Z} \lambda_{i}$, and elements $\lambda \in P$ are called coweights of $R$. A coweight $\lambda \in P$ is said to be dominant if $\left\langle\lambda, \alpha_{i}\right\rangle \geq 0$ for all $i \in I_{0}$, and we write $P^{+}$for the set of all dominant coweights. Finally, let $Q=\sum_{\alpha \in R} \mathbb{Z} \alpha^{\vee}$ be the coroot lattice of $R$, and let $Q^{+}=\sum_{\alpha \in R^{+}} \mathbb{N} \alpha^{\vee}$, where $\mathbb{N}=\{0,1,2 \ldots\}$. Note that $Q \subseteq P$.

For those readers not so familiar with the non-reduced root systems, we make the following comments. For each $n \geq 1$ there is exactly one irreducible non-reduced root system (up to isomorphism) of rank $n$, denoted by $B C_{n}$. To describe the root system $B C_{n}$, we may take $E=\mathbb{R}^{n}$ with the usual inner product, and let $R$ consist of the vectors $\pm e_{i}, \pm 2 e_{i}$ and $\pm e_{j} \pm e_{k}$ for $1 \leq i \leq n$ and $1 \leq j<k \leq n$. Let $\alpha_{j}=e_{j}-e_{j+1}$ for $1 \leq j<n$ and $\alpha_{n}=e_{n}$. Then $B=\left\{\alpha_{j}\right\}_{j=1}^{n}$ is a base of $R$, and $R^{+}$consists of the vectors $e_{i}, 2 e_{i}$ and $e_{j} \pm e_{k}$ for $1 \leq i \leq n$ and $1 \leq j<k \leq n$. The fundamental coweights are $\lambda_{i}=e_{1}+\cdots+e_{i}$ for each $1 \leq i \leq n$. Note that $R^{\vee}=R$ and $Q=P$. The subsystem $R_{1}=\{\alpha \in R \mid 2 \alpha \notin R\}$ is a root system of type $C_{n}$, and the subsystem $R_{2}=\left\{\alpha \in R \mid \frac{1}{2} \alpha \notin R\right\}$ is a root system of type $B_{n}$ (with the convention that $C_{1}=B_{1}=A_{1}$ ). 
1.2. Weyl Groups. For each $\alpha \in R$ and $k \in \mathbb{Z}$ let $H_{\alpha ; k}=\{x \in E \mid\langle x, \alpha\rangle=k\}$. We call these sets affine hyperplanes, or simply hyperplanes. For each $\alpha \in R$ and $k \in$ $\mathbb{Z}$ let $s_{\alpha ; k}$ denote the orthogonal reflection in $H_{\alpha ; k}$. Thus $s_{\alpha ; k}(x)=x-(\langle x, \alpha\rangle-k) \alpha^{\vee}$ for all $x \in E$. Write $s_{\alpha}$ in place of $s_{\alpha ; 0}, s_{i}$ in place of $s_{\alpha_{i}}$ (for $i \in I_{0}$ ), and let $s_{0}=s_{\tilde{\alpha} ; 1}$. Let $W_{0}=W_{0}(R)$ be the Weyl group of $R$, and let $W=W(R)$ be the affine Weyl group of $R$. Thus $W_{0}$ is the subgroup of $\operatorname{GL}(E)$ generated by $S_{0}=\left\{s_{i}\right\}_{i \in I_{0}}$, and $W$ is the subgroup of $\operatorname{Aff}(E)$ generated by $S=\left\{s_{i}\right\}_{i \in I}$. Both $\left(W_{0}, S_{0}\right)$ and $(W, S)$ are Coxeter systems. For subsets $J \subset I$ we write $W_{J}$ for the subgroup of $W$ generated by $\left\{s_{i}\right\}_{i \in J}$. Thus $W_{0}=W_{I \backslash\{0\}}$. Given $w \in W$, we define the length $\ell(w)$ of $w$ to be smallest $n \in \mathbb{N}$ such that $w=s_{i_{1}} \ldots s_{i_{n}}$, with $i_{1}, \ldots, i_{n} \in I$. We write $M=\left(m_{i, j}\right)_{i, j=0}^{n}$ for the Coxeter matrix of $W$. Thus $m_{i, j}$ is the order of $s_{i} s_{j}$ in $W$.

1.3. The Coxeter Complex. The Coxeter complex of $W$ is the labelled simplicial complex $\Sigma_{s}$ whose vertex set is the (automatically disjoint) union over $i \in I$ of the sets $X_{i}=\left\{w W_{I \backslash\{i\}} \mid w \in W\right\}$. If $x \in X_{i}$ we say that $x$ has type $i$, and write $\tau(x)=i$. Simplices of $\Sigma_{s}$ are subsets of the maximal simplices (or chambers), which are defined to be sets of the form $\left\{w W_{I \backslash\{i\}} \mid i \in I\right\}, w \in W$. The subscript ' $s$ ' on $\Sigma_{s}$ stands for 'simplicial'. It is also possible to think of $\Sigma_{s}$ as a chamber system $\Sigma_{c}$. Let $\Sigma_{c}$ be the set of all chambers of $\Sigma_{s}$, and for $C, C^{\prime} \in \Sigma_{c}$ and $i \in I$, declare $C \sim_{i} C^{\prime}$ if either $C=C^{\prime}$ or if all the vertices of $C$ and $C^{\prime}$ are the same, except for those of type $i$.

There is a natural geometric realisation $\Sigma$ of $\Sigma_{s}$ in the case when $R$ is irreducible (when $R$ is reducible the following construction produces a polysimplicial complex). Let $\mathcal{H}$ denote the family of hyperplanes $H_{\alpha ; k}, \alpha \in R, k \in \mathbb{Z}$, and define chambers of $\Sigma$ to be open connected components of $E \backslash \bigcup_{H \in \mathcal{H}} H$. Since $R$ is irreducible, each chamber is an open (geometric) simplex [2, V, §3, No.9, Proposition 8], and we call the extreme points of the closure of chambers vertices of $\Sigma$. We write $V(\Sigma)$ for the set of all vertices of $\Sigma$.

The choice of the base $B$ gives a natural choice of a fundamental chamber

$$
C_{0}=\left\{x \in E \mid\left\langle x, \alpha_{i}\right\rangle>0 \text { for all } i \in I_{0} \text { and }\langle x, \tilde{\alpha}\rangle<1\right\} .
$$

The vertices of $C_{0}$ are the points $\{0\} \cup\left\{\lambda_{i} / m_{i}\right\}_{i \in I_{0}}$ [2, VI, §2, No.2]. There is a natural simplicial complex structure on $\Sigma$ by taking maximal simplices to be the vertex sets of chambers of $\Sigma$, and taking simplices to be subsets of maximal simplices. Thus, following the argument in [5] Lemma 1.5], we define $\tau: V(\Sigma) \rightarrow I$ to be the unique labelling of $\Sigma$ (as a simplicial complex) such that $\tau(0)=0$ and $\tau\left(\lambda_{i} / m_{i}\right)=i$. As a labelled simplicial complex, $\Sigma$ is isomorphic to $\Sigma_{s}$.

We will henceforth simply write $\Sigma$ for the Coxeter complex of $W$. It will be clear from the context if one is to regard $\Sigma$ as a simplicial complex or as a geometric realisation in $E$.

1.4. The 'Good' Vertices and Sectors of $\Sigma$. At this stage there is really no difference between the $C_{n}$ and $B C_{n}$ cases. That is, $\Sigma_{s}\left(C_{n}\right) \equiv \Sigma_{s}\left(B C_{n}\right)$. We now introduce some additional structure that will differentiate between these two cases.

The set $P$ of coweights of $R$ is a subset of $V(\Sigma)$, and we call elements of $P$ the good vertices of $\Sigma$. Note that $P\left(C_{n}\right) \neq P\left(B C_{n}\right)$. When $R$ is reduced, $P$ is the set of more familiar special vertices of $\Sigma$ [2, VI, §2, No.2, Proposition 3].

We write $I_{P}=\{\tau(\lambda) \mid \lambda \in P\} \subseteq I$. We have $I_{P}=\left\{i \in I \mid m_{i}=1\right\}$, which shows that $0 \in I_{P}$ for all root systems, and that $I_{P}=\{0\}$ if $R$ is non-reduced 
15. Lemma 4.3]. This also shows that in the non-reduced case there are 'special' vertices which are not 'good' vertices.

We define the fundamental sector of $\Sigma$ to be the open simplicial cone

$$
\mathcal{S}_{0}=\left\{x \in E \mid\left\langle x, \alpha_{i}\right\rangle>0 \text { for all } i \in I_{0}\right\} .
$$

The sectors of $\Sigma$ are then the sets $\lambda+w\left(\mathcal{S}_{0}\right)$, where $w \in W_{0}$ and $\lambda \in P$.

1.5. The Extended Affine Weyl Group. Let $\tilde{W}=\tilde{W}(R)$ be the extended affine $W$ eyl group of $R$. That is, $\tilde{W}=W_{0} \ltimes P$. For $w \in \tilde{W}$ we define

$$
\mathcal{H}(\tilde{w})=\left\{H \in \mathcal{H} \mid H \text { separates } C_{0} \text { and } \tilde{w} C_{0}\right\},
$$

and we define the length of $\tilde{w}$ to be $\ell(\tilde{w})=|\mathcal{H}(\tilde{w})|$. For $\lambda \in P$, we write $t_{\lambda} \in \tilde{W}$ for the translation $t_{\lambda}(x)=\lambda+x$ for all $x \in E$.

Let $G=\{g \in \tilde{W} \mid \ell(g)=0\}$; this is the stabiliser of $C_{0}$ in $\tilde{W}$. We have $\tilde{W} \cong W \rtimes G$ [2] VI, $\S 2$, No.3], and furthermore $G \cong P / Q$, and so $G$ is a finite abelian group. For each $\lambda \in P$, let $W_{0 \lambda}$ denote the stabiliser of $\lambda$ in $W_{0}$. Let $w_{0}$ and $w_{0 \lambda_{i}}$ denote the longest elements of $W_{0}$ and $W_{0 \lambda_{i}}$ respectively. Then

$$
G=\left\{g_{i} \mid i \in I_{P}\right\}
$$

where $g_{0}=1$ and $g_{i}=t_{\lambda_{i}} w_{0 \lambda_{i}} w_{0}$ for $i \in I_{P} \backslash\{0\}$ [15] (4.5)].

1.6. Automorphisms. An automorphism of $\Sigma$ is a bijection $\psi$ of $E$ which maps chambers, and only chambers, to chambers, with the property that chambers $C$ and $C^{\prime}$ are adjacent if and only if $\psi(C)$ is adjacent to $\psi\left(C^{\prime}\right)$. We write $\operatorname{Aut}(\Sigma)$ for the automorphism group of $\Sigma$.

Let $D$ be the Coxeter diagram of $W$. An automorphism of $D$ is a permutation $\sigma$ of $I$ such that $m_{\sigma(i), \sigma(j)}=m_{i, j}$ for all $i, j \in I$. We write $\operatorname{Aut}(D)$ for the automorphism group of $D$.

For each $\psi \in \operatorname{Aut}(\Sigma)$ there exists a $\sigma \in \operatorname{Aut}(D)$ such that $(\tau \circ \psi)(v)=(\sigma \circ \tau)(v)$ for all $v \in V(\Sigma)$, and if $C \sim_{i} C^{\prime}$ then $\psi(C) \sim_{\sigma(i)} \psi\left(C^{\prime}\right)$ [15. Proposition 4.6]. An automorphism $\psi \in \operatorname{Aut}(\Sigma)$ is called type preserving if the associated $\sigma \in \operatorname{Aut}(D)$ is the identity. By [17 Lemma 2.2] $\psi \in \operatorname{Aut}(\Sigma)$ is type preserving if and only if $\psi \in W$.

The group $\tilde{W}$ acts on $\Sigma$ and so embeds as a subgroup of $\operatorname{Aut}(\Sigma)$. The automorphisms $\tilde{w} \in \tilde{W}$, and the associated automorphisms $\sigma \in \operatorname{Aut}(D)$, are called type rotating. We write $\operatorname{Aut}_{\mathrm{tr}}(D)$ from the subgroup of $\operatorname{Aut}(D)$ consisting of all type rotating automorphisms of $D$. The group $\operatorname{Aut}_{\text {tr }}(D)$ may be described as follows. For each $i \in I_{P}$, let $\sigma_{i}$ be the automorphism of $D$ defined by the equation $\sigma_{i} \circ \tau=\tau \circ g_{i}$. Since each $\tilde{w} \in \tilde{W}$ may be written as $\tilde{w}=w g_{i}$ where $w \in W$ and $i \in I_{P}$, 2. VI, $\S 2$, No.3], we have $\operatorname{Aut}_{\operatorname{tr}}(D)=\left\{\sigma_{i} \mid i \in I_{P}\right\}$. The group $\operatorname{Aut}_{\mathrm{tr}}(D)$ is abelian (since $G$ is), it acts simply transitively on $I_{P}$, and $\sigma_{i}(0)=i$ for each $i \in I_{P}$ (see 15. Proposition 4.7(iii)]).

Remark 1.1. In the $\tilde{A}_{n}$ case, the qualification type rotating is very natural, for $\operatorname{Aut}_{\text {tr }}(D)$ consists of the permutations $i \mapsto k+i \bmod (n+1)$ for $k=0, \ldots, n$. In the general case, the term type rotating is less natural. 
1.7. Buildings and Regularity. Let $M$ be the Coxeter matrix of $W$. Recall (4, p.76-77]) that a building of type $M$ is a nonempty simplicial complex $\mathscr{X}$ which contains a family of subcomplexes called apartments such that

(i) each apartment is isomorphic to the (simplicial) Coxeter complex of $W$,

(ii) given any two chambers of $\mathscr{X}$ there is an apartment containing both, and

(iii) given any two apartments $\mathcal{A}$ and $\mathcal{A}^{\prime}$ that contain a common chamber, there exists an isomorphism $\psi: \mathcal{A} \rightarrow \mathcal{A}^{\prime}$ fixing $\mathcal{A} \cap \mathcal{A}^{\prime}$ pointwise.

We call $\mathscr{X}$ affine when $W$ is (as we assume throughout).

It is an easy consequence of this definition that $\mathscr{X}$ is a labellable simplicial complex (with set of types I). All of the isomorphisms in the above definition may be taken to be label preserving (this ensures that the labellings of $\mathscr{X}$ and $\Sigma$ are compatible). The isomorphism in (iii) is then unique [4, p.77]. Recall (4, p.30]) that the type of a simplex $\sigma$ is the subset of $I$ consisting of the labels of the vertices of $\sigma$. If $\sigma$ has type $J \subset I$, then the cotype of $\sigma$ is $I \backslash J$.

We write $V$ for the vertex set of $\mathscr{X}$, and $\mathcal{C}$ for the set of all chambers of $\mathscr{X}$. As usual, we declare chambers $c, d \in \mathcal{C}$ to be $i$-adjacent, and write $c \sim_{i} d$, if and only if either $c=d$, or if all the vertices of $c$ and $d$ are the same, except for those of type $i$. By a gallery of type $i_{1} \cdots i_{n} \in I^{*}$ (here $I^{*}$ denotes the free monoid on $I$ ) in $\mathscr{X}$ we mean a sequence $c_{0}, \ldots, c_{n}$ of chambers such that $c_{k-1} \sim_{i_{k}} c_{k}$ and $c_{k-1} \neq c_{k}$ for $1 \leq k \leq n$. If we remove the condition that $c_{k-1} \neq c_{k}$ for each $1 \leq k \leq n$, we call the sequence $c_{0}, \ldots, c_{n}$ a pre-gallery.

We say that $\mathscr{X}$ is locally finite if both $|I|<\infty$ and $\left|\left\{d \in \mathcal{C} \mid d \sim_{i} c\right\}\right|<\infty$ for all $i \in I$ and $c \in \mathcal{C}$. We call $\mathscr{X}$ regular if for each $i \in I$ the cardinality $\left|\left\{d \in \mathcal{C} \mid d \sim_{i} c\right\}\right|$ is independent of $c \in \mathcal{C}$. In this case we define numbers $\left\{q_{i}\right\}_{i \in I}$ by $q_{i}+1=\left|\left\{d \in \mathcal{C} \mid d \sim_{i} c\right\}\right|$. The numbers $q_{i}, i \in I$, are called the parameters of the building. These parameters satisfy $q_{j}=q_{i}$ if $s_{j}=w s_{i} w^{-1}$ for some $w \in W$ (see 15. Corollary 2.2]). If $w=s_{i_{1}} \cdots s_{i_{m}} \in W$ is a reduced expression we define $q_{w}=q_{i_{1}} \cdots q_{i_{m}}$, which is independent of the particular reduced expression for $w$ (see [15, Proposition 2.1(i)] or [2, IV, §1, No.5, Proposition 5]).

To each locally finite regular affine building (of irreducible type) we associate an irreducible root system $R$ (depending on the parameter system of the building) as follows:

(i) If $\mathscr{X}$ is a regular building of type $\tilde{X}_{n}$, where $X=A$ and $n \geq 2$, or $X=B$ and $n \geq 3$, or $X=D$ and $n \geq 4$, or $X=E$ and $n=6,7$ or 8 , or $X=F$ and $n=4$, or $X=G$ and $n=2$, then we take $R=X_{n}$.

(ii) If $\mathscr{X}$ is a regular $\tilde{A}_{1}$ building with $q_{0} \neq q_{1}$, then we take $R=B C_{1}$.

(iii) If $\mathscr{X}$ is a regular $\tilde{C}_{n}$ building with $n \geq 2$ and $q_{0} \neq q_{n}$, then we take $R=B C_{n}$.

(iv) If $\mathscr{X}$ is a regular $\tilde{A}_{1}$ building with $q_{0}=q_{1}$, then we take $R=A_{1}$.

(v) If $\mathscr{X}$ is a regular $\tilde{C}_{n}$ building with $n \geq 2$ and $q_{0}=q_{n}$, then we take $R=C_{n}$.

The choices above are made to ensure that $\operatorname{Aut}_{\mathrm{tr}}(D)$ preserves the parameter system of $\mathscr{X}$; that is, for $\sigma \in \operatorname{Aut}_{\text {tr }}(D), q_{\sigma(i)}=q_{i}$ for all $i \in I$. Thus, for example, (iii) above is motivated by the general parameter system of a $\tilde{C}_{n}$ building:

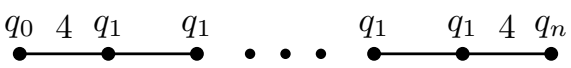


(see [15. Appendix]). If we take $R=C_{n}$, then $\operatorname{Aut}_{\mathrm{tr}}(D)=\{1, \sigma\}$, where $\sigma(0)=n$, and the condition $q_{\sigma(0)}=q_{0}$ fails. However, if $R=B C_{n}$ then $\operatorname{Aut}_{\mathrm{tr}}(D)=\{1\}$.

If $R$ is the root system associated to $\mathscr{X}$, we often say that $\mathscr{X}$ is an affine building of type $R$. Let $W_{0}, W, \tilde{W}, P, Q$, and so on be as defined earlier.

We extend the definition of $q_{w}$ made above to $\tilde{W}$ by setting $q_{\tilde{w}}=q_{w}$ if $\tilde{w}=w g$, where $w \in W$ and $g \in G$. We note that if $\ell(u v)=\ell(u)+\ell(v)$ then $q_{u v}=q_{u} q_{v}$. In particular, if $\lambda, \mu, \nu \in P^{+}$, then by [14, (2.4.1)]

$$
q_{t_{\lambda+\mu}}=q_{t_{\lambda}} q_{t_{\mu}}, \quad \text { and so } \quad q_{t_{\nu}}=\prod_{i=1}^{n} q_{t_{\lambda_{i}}}^{\left\langle\nu, \alpha_{i}\right\rangle} .
$$

Definition 1.2. Let $\mathscr{X}$ be an affine building of type $R$ with vertex set $V$, and let $\Sigma=\Sigma(R)$. Let $V_{\mathrm{sp}}(\Sigma)$ denote the set of all special vertices of $\Sigma$, and let $I_{\mathrm{sp}}=\left\{\tau(\lambda) \mid \lambda \in V_{\mathrm{sp}}(\Sigma)\right\}$. Then:

(i) A vertex $x \in V$ is said to be special if $\tau(x) \in I_{\text {sp }}$. We write $V_{\mathrm{sp}}$ for the set of all special vertices of $\mathscr{X}$.

(ii) A vertex $x \in V$ is said to be $\operatorname{good}$ if $\tau(x) \in I_{P}$. We write $V_{P}$ for the set of all good vertices of $\mathscr{X}$.

Clearly $V_{P} \subseteq V_{\mathrm{sp}}$. In fact if $R$ is reduced then $V_{P}=V_{\mathrm{sp}}$. If $R$ is non-reduced (so $R$ is of type $B C_{n}$ for some $n \geq 1$ ), then $V_{P}$ is the set of all type 0 vertices of $\mathscr{X}$, whereas $V_{\mathrm{sp}}$ is the set of all type 0 and type $n$ vertices of $\mathscr{X}$.

It is clear that a vertex $x \in V$ is good if and only if there exists an apartment $\mathcal{A}$ containing $x$ and a type preserving isomorphism $\psi: \mathcal{A} \rightarrow \Sigma$ such that $\psi(x) \in P$.

Definition 1.3. Let $\mathcal{A}$ be an apartment of $\mathscr{X}$. An isomorphism $\psi: \mathcal{A} \rightarrow \Sigma$ is called type rotating if and only if it is of the form $\psi=w \circ \psi_{0}$, where $\psi_{0}: \mathcal{A} \rightarrow \Sigma$ is a type preserving isomorphism, and $w \in \tilde{W}$. An isomorphism $\varphi: \Sigma \rightarrow \mathcal{A}$ is called type rotating if and only if $\varphi^{-1}: \mathcal{A} \rightarrow \Sigma$ is type rotating.

1.8. The Algebra $\mathscr{A}$. Let $\mathscr{X}$ be an affine building of type $R$ with parameter system $\left\{q_{i}\right\}_{i \in I}$. Given $x \in V_{P}$ and $\lambda \in P^{+}$, let $V_{\lambda}(x)$ be the set of all $y \in V_{P}$ such that there exists an apartment $\mathcal{A}$ containing $x$ and $y$, and a type rotating isomorphism $\psi: \mathcal{A} \rightarrow \Sigma$ such that $\psi(x)=0$ and $\psi(y)=\lambda$. In [15, Proposition 5.6] we showed that for each $x \in V_{P},\left\{V_{\lambda}(x)\right\}_{\lambda \in P^{+}}$forms a partition of $V_{P}$.

Remark 1.4. Let $V_{Q}$ denote the set of all type 0 vertices of $\mathscr{X}$. If $\mathscr{X}$ is the Bruhat-Tits building of a group $G$ of $p$-adic type (see the introduction), then the sets $V_{\lambda}(x), \lambda \in Q \cap P^{+}$, are the orbits in $V_{Q}=G / G_{x}$ of the isotropy group $G_{x}$.

For $\lambda \in P^{+}$, let $\lambda^{*}=-w_{0} \lambda$, where $w_{0}$ is the longest element of $W_{0}$. In 15, Proposition 5.8] we showed that $\lambda^{*} \in P^{+}$for all $\lambda \in P^{+}$, and that $y \in V_{\lambda}(x)$ if and only if $x \in V_{\lambda^{*}}(y)$. Note that $*$ is trivial unless $w_{0} \neq-1$, that is, unless $R=A_{n}, D_{2 n+1}$ or $E_{6}$ for some $n \geq 2$ (see [2, Plates I-IX]).

Let $\lambda \in P^{+}$and write $l=\tau(\lambda)$. Let $w_{\lambda}$ be the unique minimal length double coset representative of $W_{I \backslash\{0\}} t_{\lambda}^{\prime} W_{I \backslash\{l\}}$, where $t_{\lambda}^{\prime} \in W$ is (uniquely) defined by $t_{\lambda}=t_{\lambda}^{\prime} g$ for some $g \in G$ (see [15, $\left.\S 4.9\right]$ ). For finite subsets $U \subset W$ we write

$$
U(q)=\sum_{w \in U} q_{w}
$$

for the Poincaré polynomial of $U$. 
In [15] Theorem 5.15] we showed that $\left|V_{\lambda}(x)\right|=\left|V_{\lambda}(y)\right|$ for all $x, y \in V_{P}$ and $\lambda \in P^{+}$, and we denote this common value by $N_{\lambda}$. We have

$$
N_{\lambda}=\frac{W_{0}(q)}{W_{0 \lambda}(q)} q_{w_{\lambda}}=N_{\lambda^{*}},
$$

where $W_{0 \lambda}=\left\{w \in W_{0} \mid w \lambda=\lambda\right\}$. We have the following alternative formula.

Proposition 1.5. Let $\lambda \in P^{+}$. Then

$$
N_{\lambda}=\frac{W_{0}\left(q^{-1}\right)}{W_{0 \lambda}\left(q^{-1}\right)} q_{\lambda},
$$

where $W_{0}\left(q^{-1}\right)=\sum_{w \in W_{0}} q_{w}^{-1}$, and similarly for $W_{0 \lambda}\left(q^{-1}\right)$.

Proof. Let $l=\tau(\lambda)$, and let $w_{0}$ and $w_{0 \lambda}$ be the longest elements of $W_{0}$ and $W_{0 \lambda}$, respectively. Since $t_{\lambda}=w_{\lambda} g_{l} w_{0} w_{0 \lambda}$ and $\ell\left(w_{\lambda} g_{l} w_{0} w_{0 \lambda}\right)=\ell\left(w_{\lambda} g_{l}\right)+\ell\left(w_{0} w_{0 \lambda}\right)$ (see 15. Proposition 4.15]) we have $q_{t_{\lambda}}=q_{w_{\lambda}} q_{w_{0} w_{0 \lambda}}$. By [2, VI, $\S 1$, No.6, Corollary 3 to Proposition 17] we have $\ell\left(w_{0} w\right)=\ell\left(w_{0}\right)-\ell(w)$ for all $w \in W_{0}$, and it follows that $q_{w_{0} w_{0 \lambda}}=q_{w_{0}} q_{w_{0 \lambda}}^{-1}$. Thus

$$
q_{w_{\lambda}}=q_{t_{\lambda}} q_{w_{0} w_{0 \lambda}}^{-1}=q_{t_{\lambda}} q_{w_{0}}^{-1} q_{w_{0 \lambda}} .
$$

Furthermore, again using the fact that $\ell\left(w_{0} w\right)=\ell\left(w_{0}\right)-\ell(w)$ for all $w \in W_{0}$, we have

$$
W_{0}(q)=\sum_{w \in W_{0}} q_{w_{0} w}=q_{w_{0}} W_{0}\left(q^{-1}\right)
$$

and similarly, since $W_{0 \lambda}$ is a Coxeter group, $W_{0 \lambda}(q)=q_{w_{0 \lambda}} W_{0 \lambda}\left(q^{-1}\right)$.

Putting all of this together, and using (1.5), the result follows.

We say that $\lambda \in P$ is strongly dominant if $\left\langle\lambda, \alpha_{i}\right\rangle>0$ for all $i=1, \ldots, n$, and we write $P^{++}$for the set of all strongly dominant coweights. Note that when $\lambda \in P^{++}$, $W_{0 \lambda}=\{1\}$, and so by Proposition 1.5

$$
N_{\lambda}=W_{0}\left(q^{-1}\right) q_{t_{\lambda}} \quad \text { for all } \lambda \in P^{++} .
$$

For each $\lambda \in P^{+}$we define an operator $A_{\lambda}$, acting on the space of all functions $f: V_{P} \rightarrow \mathbb{C}$ by

$$
\left(A_{\lambda} f\right)(x)=\frac{1}{N_{\lambda}} \sum_{y \in V_{\lambda}(x)} f(y) \quad \text { for all } x \in V_{P}
$$

Let $\mathscr{A}$ be the linear span of $\left\{A_{\lambda}\right\}_{\lambda \in P^{+}}$over $\mathbb{C}$. For each $\lambda, \mu \in P^{+}$there exist numbers $a_{\lambda, \mu ; \nu} \in \mathbb{Q}^{+}$such that

$$
A_{\lambda} A_{\mu}=\sum_{\nu \in P^{+}} a_{\lambda, \mu ; \nu} A_{\nu} \quad \text { and } \quad \sum_{\nu \in P^{+}} a_{\lambda, \mu ; \nu}=1
$$

(see 15. Corollary 5.22]), and so $\mathscr{A}$ is an algebra. For all $\lambda, \mu, \nu \in P^{+}$we have

$$
a_{\lambda, \mu ; \nu}=\frac{N_{\nu}}{N_{\lambda} N_{\mu}}\left|V_{\lambda}(x) \cap V_{\mu^{*}}(y)\right|
$$

where $x, y \in V_{P}$ are any vertices with $y \in V_{\nu}(x)$. 
1.9. The Isomorphism $A_{\lambda} \mapsto P_{\lambda}(x)$. Let $\mathbb{C}[P]$ be the group algebra (over $\mathbb{C}$ ) of $P$, with the group operation written multiplicatively. Thus elements of $\mathbb{C}[P]$ are linear combinations of the formal exponentials $\left\{x^{\lambda}\right\}_{\lambda \in P}$. The group $W_{0}$ acts on $\mathbb{C}[P]$ by linearly extending $w x^{\lambda}=x^{w \lambda}$, and we write $\mathbb{C}[P]^{W_{0}}$ for the algebra consisting of all those $f \in \mathbb{C}[P]$ such that $w f=f$ for all $w \in W_{0}$.

Let $R_{1}=\{\alpha \in R \mid 2 \alpha \notin R\}, R_{2}=\left\{\alpha \in R \mid \frac{1}{2} \alpha \notin R\right\}$ and $R_{3}=R_{1} \cap R_{2}$ (so $R_{1}=R_{2}=R_{3}=R$ if $R$ is reduced). For $\alpha \in R_{2}$, write $q_{\alpha}=q_{i}$ if $|\alpha|=\left|\alpha_{i}\right|$, where $|\cdot|$ denotes Euclidean root length (if $|\alpha|=\left|\alpha_{i}\right|$ then necessarily $\alpha \in R_{2}$ ). Since $q_{j}=q_{i}$ whenever $s_{j}=w s_{i} w^{-1}$ for some $w \in W$, it follows that $q_{i}=q_{j}$ whenever $\left|\alpha_{i}\right|=\left|\alpha_{j}\right|$, and so the definition of $q_{\alpha}$ is unambiguous.

Note that $R=R_{3} \cup\left(R_{1} \backslash R_{3}\right) \cup\left(R_{2} \backslash R_{3}\right)$ where the union is disjoint. Define a set of numbers $\left\{\tau_{\alpha}\right\}_{\alpha \in R}$ by

$$
\tau_{\alpha}= \begin{cases}q_{\alpha} & \text { if } \alpha \in R_{3} \\ q_{0} & \text { if } \alpha \in R_{1} \backslash R_{3} \\ q_{\alpha} q_{0}^{-1} & \text { if } \alpha \in R_{2} \backslash R_{3} .\end{cases}
$$

It is convenient to define $\tau_{\alpha}=1$ if $\alpha \notin R$. Note that when $R$ is reduced, $\tau_{\alpha}=q_{\alpha}$, and expressions like $\tau_{\alpha / 2}$ and $\tau_{2 \alpha}$ for $\alpha \in R$ become 1 in subsequent formulae.

For $\lambda \in P^{+}$define the Macdonald spherical function $P_{\lambda}(x) \in \mathbb{C}[P]^{W_{0}}$ by

$$
P_{\lambda}(x)=\frac{q_{t_{\lambda}}^{-1 / 2}}{W_{0}\left(q^{-1}\right)} \sum_{w \in W_{0}} w\left(x^{\lambda} \prod_{\alpha \in R^{+}} \frac{1-\tau_{\alpha}^{-1} \tau_{\alpha / 2}^{-1 / 2} x^{-\alpha^{\vee}}}{1-\tau_{\alpha / 2}^{-1 / 2} x^{-\alpha^{\vee}}}\right) .
$$

By Proposition A.6. this formula is equivalent to [15, (6.4)].

The main theorem of [15] is the following.

Theorem 1.6. [15, Theorem 6.16] The map $A_{\lambda} \mapsto P_{\lambda}(x)$ determines an algebra isomomorphism, and so $\mathscr{A} \cong \mathbb{C}[P]^{W_{0}}$.

Remark 1.7. In [15] we obtained the isomorphism $\mathscr{A} \rightarrow \mathbb{C}[P]^{W_{0}}$ using the fact that $\mathbb{C}[P]^{W_{0}}$ is the center of an appropriately defined affine Hecke algebra. We will not need this fact here.

1.10. The Reducible Case. When $\mathscr{X}$ is a building of reducible type, then $\mathscr{X}$ has a natural description as a polysimplicial complex, and (assuming regularity) we can associate a reducible root system $R$ to $\mathscr{X}$ (bearing in mind our conventions in Section [1.7). This leads to an analogous definition of the algebra $\mathscr{A}$ in this case.

It turns out that $\mathscr{X}$ decomposes (essentially uniquely) into the cartesian product of certain irreducible components $\left\{\mathscr{X}_{j}\right\}_{j=1}^{k}$, each of which is an irreducible (regular) building. Writing $\mathscr{A}_{j}$ for the algebra of vertex set averaging operators for $\mathscr{X}_{j}$, it is not difficult to show that $\mathscr{A} \cong \mathscr{A}_{1} \times \cdots \times \mathscr{A}_{k}$, where $\times$ is direct product (see [15, Remark 0.1]). Thus knowledge of the algebra homomorphisms $h_{j}: \mathscr{A}_{j} \rightarrow \mathbb{C}$ (using the results of this paper) gives a complete description of the algebra homomorphisms $h: \mathscr{A} \rightarrow \mathbb{C}$. Thus we can restrict our attention to irreducible buildings throughout, without any loss of generality.

\section{The Macdonald Formula for the Algebra Homomorphisms}

By Theorem[1.6] the map $A_{\lambda} \mapsto P_{\lambda}(x)$ determines an isomorphism from $\mathscr{A}$ onto $\mathbb{C}[P]^{W_{0}}$. In this section we will use this result to describe the Macdonald formula for the algebra homomorphisms $h: \mathscr{A} \rightarrow \mathbb{C}$. 
For $u \in \operatorname{Hom}\left(P, \mathbb{C}^{\times}\right)$, write $u^{\lambda}$ in place of $u(\lambda)$. Each $u \in \operatorname{Hom}\left(P, \mathbb{C}^{\times}\right)$induces a homomorphism, also called $u$, on $\mathbb{C}[P]$, and all homomorphisms $\mathbb{C}[P] \rightarrow \mathbb{C}$ arise in this way. For $w \in W_{0}$ and $u \in \operatorname{Hom}\left(P, \mathbb{C}^{\times}\right)$we write $w u \in \operatorname{Hom}\left(P, \mathbb{C}^{\times}\right)$for the homomorphism $(w u)^{\lambda}=u^{w \lambda}$. If $u \in \operatorname{Hom}\left(P, \mathbb{C}^{\times}\right)$, we write $P_{\lambda}(u)$ in place of $u\left(P_{\lambda}(x)\right)$. Thus

$$
\begin{aligned}
P_{\lambda}(u) & =\frac{q_{t_{\lambda}}^{-1 / 2}}{W_{0}\left(q^{-1}\right)} \sum_{w \in W_{0}} c(w u) u^{w \lambda}, \quad \text { where } \\
c(u) & =\prod_{\alpha \in R^{+}} \frac{1-\tau_{\alpha}^{-1} \tau_{\alpha / 2}^{-1 / 2} u^{-\alpha^{\vee}}}{1-\tau_{\alpha / 2}^{-1 / 2} u^{-\alpha^{\vee}}},
\end{aligned}
$$

provided, of course, that the denominators of the $c(w u)$ functions do not vanish. Since $P_{\lambda}(u)$ is a Laurent polynomial, these singular cases can be obtained from the general formula by taking an appropriate limit (see [12 §4.6]). Finally, for $u \in \operatorname{Hom}\left(P, \mathbb{C}^{\times}\right)$, let $h_{u}: \mathscr{A} \rightarrow \mathbb{C}$ be the linear map with $h_{u}\left(A_{\lambda}\right)=P_{\lambda}(u)$ for each $\lambda \in P^{+}$.

Proposition 2.1. (cf. [12, Theorem 3.3.12])

(i) Each algebra homomorphism $h: \mathscr{A} \rightarrow \mathbb{C}$ is of the form $h=h_{u}$ for some $u \in \operatorname{Hom}\left(P, \mathbb{C}^{\times}\right)$, and

(ii) $h_{u}=h_{u^{\prime}}$ if and only if $u^{\prime}=w u$ for some $w \in W_{0}$.

Proof. Since $\mathbb{C}[P]$ is integral over $\mathbb{C}[P]^{W_{0}}$ 1, V, Exercise 12], every homomorphism $\mathbb{C}[P]^{W_{0}} \rightarrow \mathbb{C}$ is the restriction of a homomorphism $\mathbb{C}[P] \rightarrow \mathbb{C}$, and (i) follows.

It is clear that if $u^{\prime}=w u$ for some $w \in W_{0}$, then $h_{u^{\prime}}=h_{u}$. Suppose now that $h_{u}=h_{u^{\prime}}$. Since $\left\{P_{\lambda}(x)\right\}_{\lambda \in P^{+}}$forms a basis of $\mathbb{C}[P]^{W_{0}}$ we see that $u$ and $u^{\prime}$ agree on $\mathbb{C}[P]^{W_{0}}$, and thus their kernels are maximal ideals of $\mathbb{C}[P]$ lying over the same maximal ideal of $\mathbb{C}[P]^{W_{0}}$. The result now follows by [1, V, Exercise 13].

We call the formula $h_{u}\left(A_{\lambda}\right)=P_{\lambda}(u)$ the Macdonald formula for the algebra homomorphisms $\mathscr{A} \rightarrow \mathbb{C}$. By comparing Proposition A.1] and [12, Corollary 3.2.5] we see that in the case when $P=Q$ (that is, when $R$ is of type $E_{8}, F_{4}, G_{2}$ or $B C_{n}$ for some $n \geq 1$ ) our formula $P_{\lambda}(u)$ agrees with the formula in [12, Theorem 4.1.2]. The reason we require $P=Q$ here is because in 12$] u \in \operatorname{Hom}\left(Q, \mathbb{C}^{\times}\right.$) (although $u$ there is called $s$ ), which is a consequence of Macdonald requiring his group $G$ of $p$-adic type to be simply connected.

Remark 2.2. The function $P_{\lambda}(u)$ may be regarded as a function of the variables $u_{i}=u^{\lambda_{i}} \in \mathbb{C}^{\times}, i=1, \ldots, n$. However, in general the coroots $\alpha^{\vee}, \alpha \in R^{+}$, appearing in the formula for $c(u)$ do not have particularly neat expressions in terms of the basis $\left\{\lambda_{i}\right\}_{i=1}^{n}$. Thus in any given specific case it is often useful to work with numbers other than the $\left\{u_{i}\right\}_{i=1}^{n}$.

Let us illustrate this in the $R=D_{n}$ case, which may be described as follows (see 2. Plate IV]). Let $E=\mathbb{R}^{n}$ with standard orthonormal basis $\left\{e_{i}\right\}_{i=1}^{n}$, and take $R$ to be the set of vectors $\pm e_{i} \pm e_{j}, 1 \leq i<j \leq n$, where the \pm signs may be taken independently. We have $R^{\vee}=R$, and the set $\left\{e_{i}-e_{i+1}, e_{n-1}+e_{n}\right\}_{1 \leq i \leq n-1}$ forms a base of $R$. The corresponding set of positive roots is $\left\{e_{i}-e_{j}, e_{i}+e_{j}\right\}_{1 \leq i<j \leq n}$. Observe that $e_{1}=\lambda_{1}, e_{i}=\lambda_{i}-\lambda_{i-1}$ for $2 \leq i \leq n-2, e_{n-1}=\lambda_{n-1}+\lambda_{n}-\lambda_{n-2}$ and $e_{n}=\lambda_{n}-\lambda_{n-1}$. Thus, defining numbers $t_{i} \in \mathbb{C}^{\times}, i=1, \ldots, n$, by $t_{1}=u_{1}$, 


$$
\begin{gathered}
t_{i}=u_{i} u_{i-1}^{-1}(2 \leq i \leq n-2), t_{n-1}=u_{n-1} u_{n} u_{n-2}^{-1} \text { and } t_{n}=u_{n} u_{n-1}^{-1} \text {, we have } \\
c(u)=\prod_{1 \leq i<j \leq n} \frac{\left(1-q^{-1} t_{i}^{-1} t_{j}\right)\left(1-q^{-1} t_{i}^{-1} t_{j}^{-1}\right)}{\left(1-t_{i}^{-1} t_{j}\right)\left(1-t_{i}^{-1} t_{j}^{-1}\right)} .
\end{gathered}
$$

(Notice that $q_{i}=q$ for all $i \in I$, for there is only one root length).

\section{The Integral Formula for the Algebra Homomorphisms}

We begin with some background that involves only the root system $R$, which throughout is assumed to be irreducible. Define a partial order on $P$ by setting $\mu \preceq \lambda$ if $\lambda-\mu \in Q^{+}$.

We say that a subset $X \subset P$ is saturated [2, VI, $\S 1$, Exercise 23] if $\mu-i \alpha^{\vee} \in X$ for all $\mu \in X, \alpha \in R$, and all $i$ between 0 and $\langle\mu, \alpha\rangle$ inclusive. Every saturated set is stable under $W_{0}$, and for each $\lambda \in P^{+}$there is a unique saturated set, denoted $\Pi_{\lambda}$, with highest coweight $\lambda$ (that is, $\mu \preceq \lambda$ for all $\mu \in \Pi_{\lambda}$ ). We have

$$
\Pi_{\lambda}=\left\{w \mu \mid \mu \in P^{+}, \mu \preceq \lambda, w \in W_{0}\right\}
$$

(see [10, Lemma 13.4B] for example).

We recall the definition of the Bruhat order on $W$ [11, §5.9]. Let $v, w \in W$, and write $v \rightarrow w$ if $v=s_{\alpha ; k} w$ for some $\alpha \in R, k \in \mathbb{Z}$, and $\ell(v)<\ell(w)$. Declare $v \leq w$ if and only if there exists a sequence $v=w_{0} \rightarrow w_{1} \rightarrow \cdots \rightarrow w_{n}=w$. This gives the Bruhat (partial) order on $W$. We extend the Bruhat order to $\tilde{W}$ as in [14 $\S 2.3]$ by declaring $\tilde{v} \leq \tilde{w}$ if and only if $\tilde{v}=v g$ and $\tilde{w}=w g$ with $v \leq w$ in $W$ and $g \in G$.

By a sub-expression of a fixed reduced expression $s_{i_{1}} \cdots s_{i_{r}} \in W$ we mean a product of the form $s_{i_{k_{1}}} \cdots s_{i_{k_{q}}}$ where $1 \leq k_{1}<\cdots<k_{q} \leq r$. Let $w=s_{i_{1}} \cdots s_{i_{r}}$ be a fixed reduced expression for $w \in W$. By [11, Theorem 5.10], $v \leq w$ if and only $v$ can be obtained as a sub-expression of this reduced expression.

Proposition 3.1. Let $\tilde{v}, \tilde{w} \in \tilde{W}$ with $\tilde{v} \leq \tilde{w}$. If $\tilde{w}(0) \in \Pi_{\lambda}$, then $\tilde{v}(0) \in \Pi_{\lambda}$ too.

Proof. Suppose first that $\tilde{v}=s_{\alpha ; k} \tilde{w}$ with $\ell(\tilde{v})<\ell(\tilde{w})$. Then by [14, (2.3.3)] $H_{\alpha ; k}$ separates $C_{0}$ and $\tilde{w} C_{0}$, and thus $\langle\tilde{w}(0), \alpha\rangle-k$ is between 0 and $\langle\tilde{w}(0), \alpha\rangle$ (inclusive). Thus by the definition of saturated sets

$$
\tilde{v}(0)=\tilde{w}(0)-(\langle\tilde{w}(0), \alpha\rangle-k) \alpha^{\vee} \in \Pi_{\lambda},
$$

and the result clearly follows by induction.

Let $\mathscr{X}$ be an irreducible regular affine building. A sector of $\mathscr{X}$ is a subcomplex $\mathcal{S} \subset \mathscr{X}$ such that there exists an apartment $\mathcal{A}$ such that $\mathcal{S} \subset \mathcal{A}$ and a type preserving isomorphism $\psi: \mathcal{A} \rightarrow \Sigma$ such that $\psi(\mathcal{S})$ is a sector of $\Sigma$. The base vertex of $\mathcal{S}$ is $\psi^{-1}(\lambda)$, where $\lambda \in P$ is the base vertex of $\psi(\mathcal{S})$.

If $\mathcal{S}$ and $\mathcal{S}^{\prime}$ are sectors of $\mathscr{X}$ with $\mathcal{S}^{\prime} \subseteq \mathcal{S}$, then we say that $\mathcal{S}^{\prime}$ is a subsector of $\mathcal{S}$. The boundary $\Omega$ of $\mathscr{X}$ is the set of equivalence classes of sectors, where we declare two sectors equivalent if and only if they contain a common subsector. Given $x \in V_{P}$ and $\omega \in \Omega$, there exists a unique sector, denoted $\mathcal{S}^{x}(\omega)$, in the class $\omega$ with base vertex $x$ [17, Lemma 9.7].

Lemma 3.2. Let $\mathcal{S}$ be a sector in an apartment $\mathcal{A}$ of $\mathscr{X}$. There exists a unique type rotating isomorphism $\psi_{\mathcal{A}, \mathcal{S}}: \mathcal{A} \rightarrow \Sigma$ such that $\psi_{\mathcal{A}, \mathcal{S}}(\mathcal{S})=\mathcal{S}_{0}$. 
Proof. Let $x$ be the base vertex of $\mathcal{S}$, and let $\phi: \mathcal{A} \rightarrow \Sigma$ be a type preserving isomorphism. Writing $\lambda=\phi(x)$ we see that $t_{-\lambda} \circ \phi: \mathcal{A} \rightarrow \Sigma$ is a type rotating isomorphism mapping $\mathcal{S}$ to a sector of $\Sigma$ based at 0 . Thus $\left(t_{-\lambda} \circ \phi\right)(\mathcal{S})=w \mathcal{S}_{0}$ for some $w \in W_{0}$, and so $w^{-1} \circ t_{-\lambda} \circ \phi: \mathcal{A} \rightarrow \Sigma$ is a type rotating isomorphism satisfying the requirements of the lemma.

Let $\psi$ and $\psi^{\prime}$ be two such isomorphisms. It follows from [15, Lemma 5.3] that $\psi^{\prime} \circ \psi^{-1}=w$ for some $w \in W_{0}$ (it is important that $\psi$ and $\psi^{\prime}$ are type rotating here). We have $w\left(C_{0}\right)=C_{0}$, and so $w=1$ since $W$ acts simply transitively on the chambers of $\Sigma$ [4, p.142]. Thus $\psi^{\prime}=\psi$.

Given an apartment $\mathcal{A}$ and a sector $\mathcal{S}$ of $\mathcal{A}$, let $\rho_{\mathcal{A}, \mathcal{S}}: \mathscr{X} \rightarrow \mathcal{A}$ be the retraction onto $\mathcal{A}$ centered at $\mathcal{S}$ [4 pages 170-171]. This is defined as follows. Given any chamber $c$ of $\mathscr{X}$, there exists a subsector $\mathcal{S}^{\prime}$ of $\mathcal{S}$ and an apartment $\mathcal{A}^{\prime}$ such that $c$ and $\mathcal{S}^{\prime}$ are contained in $\mathcal{A}^{\prime}$ [4, page 170]. Writing $\psi_{\mathcal{A}^{\prime}}: \mathcal{A}^{\prime} \rightarrow \mathcal{A}$ for the (unique) isomorphism from building axiom (iii), we set $\rho_{\mathcal{A}, \mathcal{S}}(c)=\psi_{\mathcal{A}^{\prime}}(c)$, which is easily seen to be independent of the particular $\mathcal{S}^{\prime}$ and $\mathcal{A}^{\prime}$ chosen.

If $f=i_{1} \cdots i_{r} \in I^{*}$, the free monoid on $I$, we write $s_{f}=s_{i_{1}} \cdots s_{i_{r}} \in W$. For $\lambda \in P^{+}$let $w_{\lambda}$ be as in Section 1.8 Let $f_{\lambda} \in I^{*}$ be any (fixed) word of minimal length such that $s_{f_{\lambda}}=w_{\lambda}$.

Theorem 3.3. Let $x \in V_{P}, \omega \in \Omega$ and write $\mathcal{S}=\mathcal{S}^{x}(\omega)$. Let $\mathcal{A}$ be any apartment containing $\mathcal{S}$. Then $\left(\psi_{\mathcal{A}, \mathcal{S}} \circ \rho_{\mathcal{A}, \mathcal{S}}\right)(y) \in \Pi_{\lambda}$ for all $y \in V_{\lambda}(x)$.

Proof. It follows easily from [15. Proposition 5.2] that there exists a gallery $c_{0}, \ldots, c_{n}$ of type $\sigma_{i}\left(f_{\lambda}\right)$ from $x$ to $y$, where $i=\tau(x)$. Write $\Phi=\psi_{\mathcal{A}, \mathcal{S}} \circ \rho_{\mathcal{A}, \mathcal{S}}$. Then $\Phi\left(c_{0}\right), \ldots, \Phi\left(c_{n}\right)$ is a pre-gallery of type $f_{\lambda}$ from 0 to $\mu=\Phi(y)$. Thus there is a gallery of type $f_{\lambda}^{\prime}$, say, from 0 to $\mu$, where $s_{f_{\lambda}^{\prime}}$ is a sub-expression of $s_{f_{\lambda}}$. Thus $s_{f_{\lambda}^{\prime}} \leq s_{f_{\lambda}}$, and so $s_{f_{\lambda}^{\prime}} g_{l} \leq s_{f_{\lambda}} g_{l}$, where $l=\tau(\lambda)$. Since $\left(s_{f_{\lambda}} g_{l}\right)(0)=\lambda \in \Pi_{\lambda}$, it follows from Proposition 3.1 that $\mu^{\prime}=\left(s_{f_{\lambda}^{\prime}} g_{l}\right)(0) \in \Pi_{\lambda}$ too. Since $\Phi\left(c_{0}\right)=w C_{0}$ for some $w \in W_{0}$, we have $\Phi\left(c_{n}\right)=w s_{f_{\lambda}^{\prime}} C_{0}$, and so by considering types of vertices we have

Thus $\mu \in \Pi_{\lambda}$ by (3.1).

$$
\mu=w s_{f_{\lambda}^{\prime}}\left(g_{l}(0)\right)=w \mu^{\prime} .
$$

For each $x \in V_{P}, \omega \in \Omega$ and $\lambda \in P^{+}$, the intersection $V_{\lambda}(x) \cap \mathcal{S}^{x}(\omega)$ contains a unique vertex, denoted $v_{\lambda}^{x}(\omega) \in V_{P}$.

The coweights $h(x, y ; \omega)$ in the next theorem are the analogs of the well studied horocycle numbers of homogeneous trees.

Theorem 3.4. Let $\omega \in \Omega$ and let $x, y \in V_{P}$.

(i) Let $z \in \mathcal{S}^{x}(\omega) \cap \mathcal{S}^{y}(\omega)$ and write $z=v_{\nu}^{x}(\omega)=v_{\eta}^{y}(\omega)$. The coweight $\nu-\eta$ is independent of the particular $z \in \mathcal{S}^{x}(\omega) \cap \mathcal{S}^{y}(\omega)$ chosen. We denote this common value by $h(x, y ; \omega)$. If $\mu \in P^{+}$and $\mu-\nu \in P^{+}$then

$$
v_{\mu}^{x}(\omega)=v_{\mu-h(x, y ; \omega)}^{y}(\omega) .
$$

(ii) Suppose that $y \in V_{\lambda}(x)$. Write $\mathcal{S}=\mathcal{S}^{x}(\omega)$ and let $\mathcal{A}$ be any apartment containing $\mathcal{S}$. Then $h(x, y ; \omega)=\left(\psi_{\mathcal{A}, \mathcal{S}} \circ \rho_{\mathcal{A}, \mathcal{S}}\right)(y) \in \Pi_{\lambda}$.

Proof. (i) We have $v_{\nu+\mu^{\prime}}^{x}(\omega)=v_{\eta+\mu^{\prime}}^{y}(\omega)$ for all $\mu^{\prime} \in P^{+}$, since both are equal to $v_{\mu^{\prime}}^{z}(\omega)$. Thus, writing $\mu=\mu^{\prime}+\nu$ we have

$$
v_{\mu}^{x}(\omega)=v_{\mu-(\nu-\eta)}^{y}(\omega) \quad \text { whenever } \mu-\nu \in P^{+} .
$$


If we instead choose $z^{\prime} \in \mathcal{S}^{x}(\omega) \cap \mathcal{S}^{y}(\omega)$, where $z^{\prime}=v_{\nu^{\prime}}^{x}(\omega)=v_{\eta^{\prime}}^{y}(\omega)$, then following the above we have

$$
v_{\mu}^{x}(\omega)=v_{\mu-\left(\nu^{\prime}-\eta^{\prime}\right)}^{y}(\omega) \quad \text { whenever } \mu-\nu^{\prime} \in P^{+} .
$$

By choosing $\mu \in P^{+}$such that both $\mu-\nu$ and $\mu-\nu^{\prime}$ are dominant, it follows from (3.2) and (3.3) that $\nu-\eta=\nu^{\prime}-\eta^{\prime}$. Then (3.2) proves the final claim.

(ii) Write $\mu=\left(\psi_{\mathcal{A}, \mathcal{S}} \circ \rho_{\mathcal{A}, \mathcal{S}}\right)(y)$. By [4, page 170] there exists a subsector $\mathcal{S}^{\prime}$ of $\mathcal{S}=\mathcal{S}^{x}(\omega)$ such that $\mathcal{S}^{\prime}$ and $y$ lie in a common apartment $\mathcal{A}^{\prime}$. The restriction of $\rho_{\mathcal{A}, \mathcal{S}}$ to $\mathcal{A}^{\prime}$ is thus a type preserving isomorphism. Pick $\nu \in P^{+}$such that $v_{\nu}^{x}(\omega) \in \mathcal{S}^{\prime}$, $\nu-\mu \in P^{+}$and $v_{\nu}^{x}(\omega) \in \mathcal{S}^{y}(\omega)$. The map $\psi=t_{-\mu} \circ \psi_{\mathcal{A}, \mathcal{S}} \circ \rho_{\mathcal{A}, \mathcal{S}}: \mathcal{A}^{\prime} \rightarrow \Sigma$ is a type rotating isomorphism such that $\psi(y)=0$ and $\psi\left(v_{\nu}^{x}(\omega)\right)=\nu-\mu \in P^{+}$. Thus $v_{\nu}^{x}(\omega) \in V_{\nu-\mu}(y) \cap \mathcal{S}^{y}(\omega)$, and so $h(x, y ; \omega)=\mu$. The fact that $h(x, y ; \omega) \in \Pi_{\lambda}$ now follows from Theorem 3.3 .

Proposition 3.5. For all $x, y, z \in V_{P}$ and $\omega \in \Omega$ we have the 'cocycle relation' $h(x, y ; \omega)=h(x, z ; \omega)+h(z, y ; \omega)$. Thus $h(x, x ; \omega)=0$ and $h(y, x ; \omega)=-h(x, y ; \omega)$.

Proof. For $\mu=k_{1} \lambda_{1}+\cdots+k_{n} \lambda_{n} \in P^{+}$with each $k_{i}$ sufficiently large we have

$$
v_{\mu}^{x}(\omega)=v_{\mu-h(x, z ; \omega)}^{z}(\omega)=v_{\mu-h(x, z ; \omega)-h(z, y ; \omega)}^{y}(\omega)
$$

and the result follows.

The following theorem shows that if $y \in V_{\lambda}(x)$, then for any $\omega \in \Omega, \mathcal{S}^{y}(\omega)$ contains all vertices $v_{\mu}^{x}(\omega)$ for $\mu \in P^{+}$large enough, where large enough depends only on $\lambda$, not on the particular $x, y$ and $\omega$.

For $\lambda \in P^{+}$, write $\mu \gg \lambda$ to mean that $\mu-\Pi_{\lambda} \subset P^{+}$(in particular, $\mu \in P^{+}$).

Theorem 3.6. Let $x \in V_{P}, \lambda \in P^{+}$and $y \in V_{\lambda}(x)$. Then $v_{\mu}^{x}(\omega) \in \mathcal{S}^{y}(\omega)$ for all $\omega \in \Omega$ and all $\mu \gg \lambda$, and so $v_{\mu}^{x}(\omega)=v_{\mu-h(x, y ; \omega)}^{y}(\omega)$ for all $\omega \in \Omega$ and all $\mu \gg \lambda$.

Remark 3.7. The proof of Theorem 3.6 will be given after the following preliminary results. We are thankful to an anonymous referee for sketching this proof of the present form of Theorem 3.6] which replaces our less sharp version of this result.

Lemma 3.8. Suppose that $\mathcal{A}_{1}$ and $\mathcal{A}_{2}$ are apartments containing a common sector $\mathcal{S}$. Then the maps $\left.\rho_{\mathcal{A}_{1}, \mathcal{S}}\right|_{\mathcal{A}_{2}}: \mathcal{A}_{2} \rightarrow \mathcal{A}_{1}$ and $\left.\rho_{\mathcal{A}_{2}, \mathcal{S}}\right|_{\mathcal{A}_{1}}: \mathcal{A}_{1} \rightarrow \mathcal{A}_{2}$ are mutually inverse isomorphisms which fix $\mathcal{A}_{1} \cap \mathcal{A}_{2}$ pointwise.

Proof. Fix any chamber $c \subset \mathcal{S}$, and let $\varphi: \mathcal{A}_{1} \rightarrow \mathcal{A}_{2}$ be the unique isomorphism fixing $\mathcal{A}_{1} \cap \mathcal{A}_{2}$ pointwise. Then by definition we have $\left.\rho_{\mathcal{A}_{2}, \mathcal{S}}\right|_{\mathcal{A}_{1}}=\varphi$, and since $\varphi^{-1}: \mathcal{A}_{2} \rightarrow \mathcal{A}_{1}$ is the unique isomorphism fixing $\mathcal{A}_{1} \cap \mathcal{A}_{2}$ pointwise we have $\left.\rho_{\mathcal{A}_{1}, \mathcal{S}}\right|_{\mathcal{A}_{2}}=\varphi^{-1}$.

Lemma 3.9. Let $\mathcal{A}$ be an apartment in $\mathscr{X}$, let $\mathcal{S}$ be a sector in $\mathcal{A}$, and let $H$ be a wall in $\mathcal{A}$. Then exactly one of the two closed half-apartments in $\mathcal{A}$ determined by $H, H^{+}$say, contains a subsector of $\mathcal{S}$. If $x \in V_{P} \cap H^{+}$, then the sector based at $x$ and equivalent to $\mathcal{S}$ is contained in $\mathrm{H}^{+}$.

Proof. Let $\psi=\psi_{\mathcal{A}, \mathcal{S}}$ (see Lemma 3.2). Then $\psi(H)=H_{\alpha ; k}$ for some $\alpha \in R$ and $k \in \mathbb{Z}$. Since $H_{-\alpha ; k}=H_{\alpha ;-k}$, we may suppose that $\alpha \in R^{+}$. If $k \leq 0$, then $\mathcal{S}_{0} \subset H_{\alpha ; k}^{+}=\{x:\langle x, \alpha\rangle \geq k\}$. If $k \geq 1$, then $\lambda+\mathcal{S}_{0} \subset H_{\alpha ; k}^{+}$for $\lambda=k\left(\lambda_{1}+\cdots+\lambda_{n}\right)$.

The final statement follows from [17, Lemma 9.1]. 
Proposition 3.10. Let $c_{0}, \ldots, c_{m}$ be a gallery of type $j_{1} \cdots j_{m}$, and let $\omega \in \Omega$. Let $\mathcal{A}$ be an apartment containing $c_{0}$ and a sector $\mathcal{S}$ in the class $\omega$. Let $\rho=\rho_{\mathcal{S}, \mathcal{A}}$, and let $e_{k}=\rho\left(c_{k}\right)$ for $k=0, \ldots, m$. For $k=1, \ldots, m$, let $H_{k}$ denote the wall in $\mathcal{A}$ containing the panel in $e_{k-1}$ and in $e_{k}$ of type $j_{k}$. Let $H_{k}^{+}$denote the half-apartment in $\mathcal{A}$ bounded by $H_{k}$ which contains a subsector of $\mathcal{S}$ (see Lemma 3.9).

Then there exists an apartment $\mathcal{B}$ containing $c_{m}$ and

$$
\bigcap_{k=1}^{m} H_{k}^{+}
$$

(and therefore $\mathcal{B}$ contains a sector in the class $\omega$ ).

Proof. By induction on $m$. If $m=1$, the panel of cotype $j_{1}$ common to $e_{0}=c_{0}$ and $e_{1}$ is contained in $c_{1}$ and in $H_{1}$. So by the proof of [17. Lemma 9.4], there is an apartment $\mathcal{B}$ containing $H_{1}^{+}$and $c_{1}$.

Now suppose that $m>1$ and that there is an apartment $\mathcal{B}^{\prime}$ containing $c_{m-1}$ and $\bigcap_{i=1}^{m-1} H_{k}^{+}$. Let $\mathcal{S}^{\prime}$ be any sector in the class $\omega$ contained in $\bigcap_{k=1}^{m-1} H_{k}^{+}$, and let $\rho^{\prime}=\rho_{\mathcal{S}^{\prime}, \mathcal{B}^{\prime}}$. If $\mathcal{T}$ is a common subsector of $\mathcal{S}$ and $\mathcal{S}^{\prime}$, then $\rho=\rho_{\mathcal{T}, \mathcal{A}}$ and $\rho^{\prime}=\rho_{\mathcal{T}, \mathcal{B}^{\prime}}$. So by Lemma 3.8 the maps $\left.\rho\right|_{\mathcal{B}^{\prime}}: \mathcal{B}^{\prime} \rightarrow \mathcal{A}$ and $\left.\rho^{\prime}\right|_{\mathcal{A}}: \mathcal{A} \rightarrow \mathcal{B}^{\prime}$ are mutually inverse isomorphisms.

Let $H$ denote the wall in $\mathcal{B}^{\prime}$ containing the panel of cotype $j_{m}$ in $c_{m-1}$ and in $c_{m}$, and let $H^{+}$denote the half-apartment in $\mathcal{B}^{\prime}$ bounded by $H$ and containing a subsector of $\mathcal{S}$. The half-apartment $\rho^{\prime}\left(H_{m}^{+}\right)$in $\mathcal{B}^{\prime}$ is bounded by $H$. To see this, let $\pi$ denote the panel in $c_{m-1}$ and $c_{m}$ of cotype $j_{m}$, and let $\pi^{\prime}$ denote the panel in $e_{m-1}=\rho\left(c_{m-1}\right)$ and $e_{m}=\rho\left(c_{m}\right)$ of cotype $j_{m}$. Then $\pi^{\prime}=\rho\left(c_{m-1} \cap c_{m}\right)$, and so $\rho^{\prime}\left(\pi^{\prime}\right)=c_{m-1} \cap c_{m}=\pi$. Now $\pi^{\prime}$ is in the wall of $\mathcal{A}$ bounding $H_{m}^{+}$, and so $\pi=\rho^{\prime}\left(\pi^{\prime}\right)$ is in the wall of $\mathcal{B}$ bounding $\rho^{\prime}\left(H_{m}^{+}\right)$. But $\pi$ is in the wall $H$ of $\mathcal{B}$ bounding $H^{+}$. Furthermore, $\rho^{\prime}\left(H_{m}^{+}\right)$contains a subsector of $\mathcal{S}$, because $H_{m}^{+} \cap \mathcal{B}^{\prime}$ contains such a subsector, and is fixed by $\rho^{\prime}$. Thus

$$
\rho^{\prime}\left(H_{m}^{+}\right)=H^{+} .
$$

By the proof of [17, Lemma 9.4], there is an apartment $\mathcal{B}$ containing $c_{m}$ and $H^{+}$. Since $H_{1}^{+} \cap \cdots \cap H_{m-1}^{+}$is contained in $\mathcal{B}^{\prime}$, it is fixed by $\rho^{\prime}$, and so

$$
H_{1}^{+} \cap \cdots \cap H_{m}^{+}=\rho^{\prime}\left(\left(H_{1}^{+} \cap \cdots \cap H_{m-1}^{+}\right) \cap H_{m}^{+}\right) \subset \rho^{\prime}\left(H_{m}^{+}\right) .
$$

Thus by (3.4), $H_{1}^{+} \cap \cdots \cap H_{m}^{+} \subset H^{+} \subset \mathcal{B}$, completing the induction step.

Lemma 3.11. Suppose that $C_{0}=D_{0}, \ldots, D_{m}$ is a gallery in $\Sigma$ joining 0 to $\lambda$ of minimal length, and that $C_{0}=E_{0}, \ldots, E_{m}$ is a pre-gallery in $\Sigma$. If both the gallery and the pre-gallery have the same type, then each type $l=\tau(\lambda)$ vertex of any of the $D_{i}$ 's and $E_{i}$ 's is in $\Pi_{\lambda}$.

Proof. For $k=0, \ldots, m$, define $u_{k}, v_{k} \in W$ by $D_{k}=u_{k} C_{0}$ and $E_{k}=v_{k} C_{0}$. Then the type $l$ vertices of $D_{k}$ and $E_{k}$ are $u_{k} g_{l}(0)$ and $v_{k} g_{l}(0)$ respectively (see the proof of Theorem 3.3). In particular, $u_{m} g_{l}(0)=\lambda \in \Pi_{\lambda}$.

Since the gallery $C_{0}=D_{0}, \ldots, D_{m}$ and the pre-gallery $C_{0}=E_{0}, \ldots, E_{m}$ have the same type, $j_{1} \ldots j_{m}$, say, each $u_{k}$ and each $v_{k}$ is a subexpression of the reduced expression $u_{m}=s_{j_{1}} \cdots s_{j_{m}}$. Thus $u_{k} g_{l}, v_{k} g_{l} \leq u_{m} g_{l}$ (with respect to the Bruhat order on $\tilde{W}$ ) for each $k=0, \ldots, m$.

The result now follows from Proposition 3.1 
Corollary 3.12. Suppose that $x, y \in V_{P}, \omega \in \Omega, \lambda \in P^{+}$, and $y \in V_{\lambda}(x)$. Consider a minimal gallery $c_{0}, \ldots, c_{m}$ from $x$ to $y$ and an apartment $\mathcal{A}$ containing $c_{0}$ and $a$ sector in the class $\omega$ (and hence containing $\mathcal{S}^{x}(\omega)$ ). Let $\psi: \mathcal{A} \rightarrow \Sigma$ be the typerotating isomorphism mapping $\mathcal{S}^{x}(\omega)$ to $\mathcal{S}_{0}$ (see Lemma 3.2). Finally, let $H_{i}$ and $H_{i}^{+}$be as in Proposition 3.10 and write $\rho=\rho_{\mathcal{A}, \mathcal{S}^{x}}(\omega)$. Then:

(i) $\psi^{-1}\left(\Pi_{\lambda}\right)$ contains all the type $j=\tau(y)$ vertices in each $\rho\left(c_{i}\right)$, and so in particular it contains $y^{\prime}=\rho(y)$.

(ii) If $\mu \gg \lambda$, then $v_{\mu}^{x}(\omega) \in \bigcap_{i=1}^{m} H_{i}^{+}$.

Proof. (i) Let $e_{i}=\rho\left(c_{i}\right)$ and $E_{i}=\psi\left(e_{i}\right)$ for $i=0, \ldots, m$. Let $\rho^{\prime}=\rho_{c_{0}, \mathcal{A}}$ denote the retraction of center $c_{0}$ onto $\mathcal{A}$ (see [4, $\S$ IV.3]). Let $d_{i}=\rho^{\prime}\left(c_{i}\right)$ and $D_{i}=\psi\left(d_{i}\right)$ for $i=0, \ldots, m$. Then the gallery $C_{0}=D_{0}, \ldots, D_{m}$ and the pre-gallery $C_{0}=$ $E_{0}, \ldots, E_{m}$ satisfy the hypotheses of Lemma 3.11 and the result follows.

(ii) For $i=0, \ldots, m$, let $v_{i}$ be the type $j$ vertex of $e_{i}$, and so $\left\{v_{i}\right\}_{i=0}^{m} \subset \psi^{-1}\left(\Pi_{\lambda}\right)$.

Let $1 \leq i \leq m$. If $v_{i-1}=v_{i}$, then $\psi\left(v_{i}\right) \in \Pi_{\lambda} \cap \psi\left(H_{i}\right)$, and so $\psi\left(v_{i}\right)+\mathcal{S}_{0}$ is contained in $\psi\left(H_{i}\right)^{+}$by Lemma 3.9 (here $\psi\left(H_{i}\right)^{+}$is the half-space of $\Sigma$ bounded by $\psi\left(H_{i}\right)$ and containing a subsector of $\left.\mathcal{S}_{0}=\psi\left(\mathcal{S}^{x}(\omega)\right)\right)$. Hence $\mathcal{S}^{v_{i}}(\omega)$ is contained in $H_{i}^{+}$. By our hypothesis, $\mu-\Pi_{\lambda} \subset P^{+}$, and so $\mu=\psi\left(v_{i}\right)+\nu_{i}$ for some $\nu_{i} \in P^{+}$. Hence $\psi\left(v_{\mu}^{x}(\omega)\right)=\mu \in \psi\left(v_{i}\right)+\mathcal{S}_{0} \subset \psi\left(H_{i}\right)^{+}$. Hence $v_{\mu}^{x}(\omega) \in H_{i}^{+}$.

If $v_{i-1} \neq v_{i}$, then $\psi\left(v_{i-1}\right)$ and $\psi\left(v_{i}\right)$ lie on opposite sides of $\psi\left(H_{i}\right)$. Then by Lemma 3.9 either $\psi\left(v_{i-1}\right)+\mathcal{S}_{0}$ or $\psi\left(v_{i}\right)+\mathcal{S}_{0}$ is contained in $\psi\left(H_{i}\right)^{+}$. Let us assume that $\psi\left(v_{i}\right)+\mathcal{S}_{0} \subset \psi\left(H_{i}\right)^{+}$. Since $\mu-\Pi_{\lambda} \subset P^{+}$, we can write $\mu=\psi\left(v_{i}\right)+\nu_{i}$ for some $\nu_{i} \in P^{+}$. Hence $\psi\left(v_{\mu}^{x}(\omega)\right)=\mu \in \psi\left(v_{i}\right)+\mathcal{S}_{0} \subset \psi\left(H_{i}\right)^{+}$, and so again $v_{\mu}^{x}(\omega) \in H_{i}^{+}$.

Proof of Theorem 3.6. Let $c_{0}, \ldots, c_{m}$ be a minimal gallery from $x$ to $y$, and let $\mathcal{A}, \mathcal{B}$ and $H_{1}^{+}, \ldots, H_{m}^{+}$be as in Proposition 3.10 .

By Lemma 3.8 the map $\varphi=\left.\rho_{\mathcal{A}, \mathcal{S}^{x}(\omega)}\right|_{\mathcal{B}}: \mathcal{B} \rightarrow \mathcal{A}$ is an isomorphism. It maps $y$ to $y^{\prime}=\rho_{\mathcal{A}, \mathcal{S}^{x}(\omega)}(y)$ and fixes $\mathcal{A} \cap \mathcal{B} \supset \bigcap_{i=1}^{m} H_{i}^{+}$, which contains a sector in the class $\omega$. Hence $\varphi$ maps $\mathcal{S}^{y}(\omega)$ to $\mathcal{S}^{y^{\prime}}(\omega)$. Moreover, if $\mu \gg \lambda$, then by Corollary 3.12(ii), $\varphi$ fixes $v_{\mu}^{x}(\omega)$.

Let $\psi: \mathcal{A} \rightarrow \Sigma$ be the type-rotating isomorphism mapping $\mathcal{S}^{x}(\omega)$ to $\mathcal{S}_{0}$. Then by Corollary 3.12(i) we have $y^{\prime} \in \psi^{-1}\left(\Pi_{\lambda}\right)$, and so we can write $\mu=\psi\left(y^{\prime}\right)+\nu$ for some $\nu \in P^{+}$. Therefore $v_{\mu}^{x}(\omega) \in \mathcal{S}^{y^{\prime}}(\omega)$, and applying $\varphi$, we see that $v_{\mu}^{x}(\omega) \in \mathcal{S}^{y}(\omega)$. So $v_{\mu}^{x}(\omega)=v_{\mu-h(x, y ; \omega)}^{y}(\omega)$ by the definition of $h(x, y ; \omega)$.

Let $\leq$ denote the partial order on $P^{+}$given by $\mu \leq \lambda$ if and only if $\lambda-\mu \in P^{+}$. Fixing $x \in V_{P}$, there is a natural map $\theta: \Omega \rightarrow \prod_{\lambda \in P^{+}} V_{\lambda}(x)$, where one maps $\omega$ to $\left(v_{\lambda}^{x}(\omega)\right)_{\lambda \in P^{+}}$. For each pair $\lambda, \mu \in P^{+}$with $\mu \leq \lambda$, let $\varphi_{\mu, \lambda}: V_{\lambda}(x) \rightarrow V_{\mu}(x)$ be the map $y \mapsto v_{\mu}(x, y)$, where $v_{\mu}(x, y)$ is the unique vertex in $V_{\mu}(x) \cap \operatorname{conv}\{x, y\}$ (see Appendix $\mathrm{B}$ ). Then $\left(V_{\lambda}(x), \varphi_{\mu, \lambda}\right)$ is an inverse system of topological spaces (where each finite set $V_{\lambda}(x)$ is given the discrete topology). The inverse limit $\lim _{\operatorname{ab}}\left(V_{\lambda}(x), \varphi_{\mu, \lambda}\right)$ is a compact Hausdorff topological space [3. I.9.6], and the map $\theta$ is a bijection of $\Omega$ onto this inverse limit, thus inducing a compact Hausdorff topology on $\Omega$, which we show in Theorem 3.17 is independent of $x \in V_{P}$. See Appendix B for a sketch of the proof that $\theta$ is a bijection of $\Omega$ onto $\lim _{(}\left(V_{\lambda}(x), \varphi_{\mu, \lambda}\right)$.

With $x \in V_{P}$ fixed as above, for each $y \in V_{P}$ let $\Omega_{x}\left(\overleftarrow{y)}=\left\{\omega \in \Omega \mid y \in \mathcal{S}^{x}(\omega)\right\}\right.$. The sets $\Omega_{x}(y), y \in V_{P}$, form a basis of open and closed sets for the topology on $\Omega$, and the functions $\omega \mapsto h(x, y ; \omega)$ are locally constant on $\Omega$, as we see in Lemma 3.13. 
To each $x \in V_{P}$ there is a unique regular Borel probability measure $\nu_{x}$ on $\Omega$ such that $\nu_{x}\left(\Omega_{x}(y)\right)=N_{\lambda}^{-1}$ if $y \in V_{\lambda}(x)$. To see this, for each $\lambda \in P^{+}$let $\mathscr{C}_{\lambda}(\Omega)$ be the space of all functions $f: \Omega \rightarrow \mathbb{C}$ which are constant on each set $\Omega_{x}(y), y \in V_{\lambda}(x)$. For each $\lambda \in P^{+}$define $J_{\lambda}: \mathscr{C}_{\lambda}(\Omega) \rightarrow \mathbb{C}$ by $J_{\lambda}(f)=\frac{1}{N_{\lambda}} \sum_{y \in V_{\lambda}(x)} c_{y}(f)$, where $c_{y}(f)$ is the constant value $f$ takes on $\Omega_{x}(y)$. The space of all locally constant functions $f: \Omega \rightarrow \mathbb{C}$ is $\mathscr{C}_{\infty}(\Omega)=\bigcup_{\lambda \in P^{+}} \mathscr{C}_{\lambda}(\Omega)$. Define $J: \mathscr{C}_{\infty}(\Omega) \rightarrow \mathbb{C}$ by $J(f)=J_{\lambda}(f)$ if $f \in \mathscr{C}_{\lambda}(\Omega)$. The map $J$ is linear, maps $1 \in \mathscr{C}_{\infty}(\Omega)$ to $1 \in \mathbb{C}$ and satisfies $|J(f)| \leq\|f\|_{\infty}$ for all $f \in \mathscr{C}_{\infty}(\Omega)$. Since $\mathscr{C}_{\infty}(\Omega)$ is dense in $\mathscr{C}(\Omega), J$ extends uniquely to a linear map $\tilde{J}: \mathscr{C}(\Omega) \rightarrow \mathbb{C}$ such that $|\tilde{J}(f)| \leq\|f\|_{\infty}$ for all $f \in \mathscr{C}(\Omega)$ (here $\mathscr{C}(\Omega)$ is the space of all continuous functions $f: \Omega \rightarrow \mathbb{C}$ ). Thus by the Riesz Representation Theorem there exists a unique regular Borel probability measure $\nu_{x}$ such that

$$
\tilde{J}(f)=\int_{\Omega} f(w) d \nu_{x}(\omega) \text { for all } f \in \mathscr{C}(\Omega) .
$$

In particular, $N_{\lambda}^{-1}=\nu_{x}\left(\Omega_{x}(y)\right)$ if $y \in V_{\lambda}(x)$.

In Theorem 3.17 (ii) we show that for $x, y \in V_{P}$, the measures $\nu_{x}$ and $\nu_{y}$ are mutually absolutely continuous, and we compute the Radon-Nikodym derivative.

Lemma 3.13. Let $y \in V_{\nu}(x)$ and suppose that $z \in V_{\lambda}(x) \cap V_{\mu}(y)$ with $\lambda \gg \nu$. Then

(i) $\Omega_{x}(z) \subset \Omega_{y}(z)$, and

(ii) $h(x, y ; \omega)=\lambda-\mu$ for all $\omega \in \Omega_{x}(z)$.

Proof. (i) Let $\omega \in \Omega_{x}(z)$, and so $z=v_{\lambda}^{x}(\omega)$, and by Theorem 3.6 $z \in \mathcal{S}^{y}(\omega)$. Thus $\omega \in \Omega_{y}(z)$ and so $\Omega_{x}(z) \subset \Omega_{y}(z)$. Note that if $\mu \gg \nu^{*}$ too, then $\Omega_{x}(z)=\Omega_{y}(z)$.

Since $\Omega_{x}(z) \subset \Omega_{y}(z)$ and $z \in V_{\lambda}(x) \cap V_{\mu}(y)$ we have $v_{\lambda}^{x}(\omega)=z=v_{\mu}^{y}(\omega)$ for all $\omega \in \Omega_{x}(z)$, and so (ii) follows from Theorem 3.4 (i).

Lemma 3.14. Let $x, y \in V_{P}$. In the notation of Appendix $B$, if $z \in \operatorname{conv}\{x, y\}$, then $\Omega_{x}(y) \subset \Omega_{x}(z)$.

Proof. Let $\omega \in \Omega_{x}(y)$. Then the sector $\mathcal{S}^{x}(\omega)$ contains $x$ and $y$, and hence $z$. Thus $\omega \in \Omega_{x}(z)$.

Recall that we write $P^{++}$for the set of all strongly dominant coweights of $R$, that is, those $\lambda \in P$ such that $\left\langle\lambda, \alpha_{i}\right\rangle>0$ for all $i \in I_{0}$.

Proposition 3.15. Let $\mu \in P$ be fixed. For all $\lambda \in P^{++}$such that $\lambda-\mu \in P^{++}$ we have

$$
\frac{N_{\lambda}}{N_{\lambda-\mu}}=\prod_{i=1}^{n} q_{t_{\lambda_{i}}}^{\left\langle\mu, \alpha_{i}\right\rangle}=\prod_{\alpha \in R^{+}} \tau_{\alpha}^{\langle\mu, \alpha\rangle}
$$

Proof. By (1.6) we have $N_{\lambda} N_{\lambda-\mu}^{-1}=q_{t_{\lambda}} q_{t_{\lambda-\mu}}^{-1}$, and so by (1.4)

$$
\frac{N_{\lambda}}{N_{\lambda-\mu}}=\frac{q_{t_{\lambda}}}{q_{t_{\lambda-\mu}}}=\prod_{i=1}^{n} q_{t_{\lambda_{i}}}^{\left\langle\lambda, \alpha_{i}\right\rangle-\left\langle\lambda-\mu, \alpha_{i}\right\rangle}=\prod_{i=1}^{n} q_{t_{\lambda_{i}}}^{\left\langle\mu, \alpha_{i}\right\rangle},
$$

proving the first equality. On the other hand, by Proposition A.1

$$
\frac{N_{\lambda}}{N_{\lambda-\mu}}=\frac{q_{t_{\lambda}}}{q_{t_{\lambda-\mu}}}=\prod_{\alpha \in R^{+}} \tau_{\alpha}^{\langle\lambda, \alpha\rangle-\langle\lambda-\mu, \alpha\rangle}=\prod_{\alpha \in R^{+}} \tau_{\alpha}^{\langle\mu, \alpha\rangle} .
$$


Lemma 3.16. Let $\lambda, \mu \in P^{+}$. Then $\Pi_{\lambda}+\Pi_{\mu} \subseteq \Pi_{\lambda+\mu}$.

Proof. Let $\lambda^{\prime} \in \Pi_{\lambda}$ and $\mu^{\prime} \in \Pi_{\mu}$. Then

$$
w\left(\lambda^{\prime}+\mu^{\prime}\right)=w \lambda^{\prime}+w \mu^{\prime} \preceq \lambda+\mu \quad \text { for all } w \in W_{0} .
$$

By choosing $w \in W_{0}$ such that $w\left(\lambda^{\prime}+\mu^{\prime}\right) \in P^{+}$we have $w\left(\lambda^{\prime}+\mu^{\prime}\right) \in \Pi_{\lambda+\mu}$ by (3.1), and so $\lambda^{\prime}+\mu^{\prime} \in \Pi_{\lambda+\mu}$.

Theorem 3.17. Consider the topologies and measures defined above on $\Omega$. Then

(i) The topology on $\Omega$ does not depend on the particular $x \in V_{P}$.

(ii) For $x, y \in V_{P}$, the measures $\nu_{x}$ and $\nu_{y}$ are mutually absolutely continuous, and the Radon-Nikodym derivative is given by

$$
\frac{d \nu_{y}}{d \nu_{x}}(\omega)=\prod_{i=1}^{n} q_{t_{\lambda_{i}}}^{\left\langle h(x, y ; \omega), \alpha_{i}\right\rangle}=\prod_{\alpha \in R^{+}} \tau_{\alpha}^{\langle h(x, y ; \omega), \alpha\rangle} .
$$

Proof. (i) Let $x, y \in V_{P}$, with $y \in V_{\nu}(x)$, say, and choose $\lambda \gg \nu+\nu^{*}$. Notice that this implies that $\lambda \gg \nu$. Furthermore, for each $\nu^{\prime} \in \Pi_{\nu}$, using Lemma 3.16 we have

$$
\left(\lambda-\nu^{\prime}\right)-\Pi_{\nu^{*}} \subseteq \lambda-\Pi_{\nu}-\Pi_{\nu^{*}} \subseteq \lambda-\Pi_{\nu+\nu^{*}},
$$

and so $\lambda-\nu^{\prime} \gg \nu^{*}$.

Let $\omega_{0} \in \Omega_{x}(v)$, a basic open set for the topology using $x$, where $v \in V_{\eta}(x)$, say. Since $\lambda \gg \nu$, by Theorem [3.6] we have $v_{\lambda}^{x}\left(\omega_{0}\right) \in \mathcal{S}^{y}\left(\omega_{0}\right)$. Let $z=v_{\lambda}^{x}\left(\omega_{0}\right)=$ $v_{\lambda-h\left(x, y ; \omega_{0}\right)}^{y}\left(\omega_{0}\right)$, and so $z \in V_{\lambda}(x) \cap V_{\lambda-h\left(x, y ; \omega_{0}\right)}(y)$. Now $h\left(x, y ; \omega_{0}\right) \in \Pi_{\nu}$ (see Theorem 3.4(ii)), and so by the above, $\lambda-h\left(x, y ; \omega_{0}\right) \gg \nu^{*}$. Since $\lambda \gg \nu$ too, by Lemma [3.13] we have $\Omega_{x}(z)=\Omega_{y}(z)$. Choosing $\lambda$ so that $\lambda-\eta \in P^{+}$we have $\Omega_{x}(z) \subset \Omega_{x}(v)$ by Lemma 3.14 Thus, since $z=v_{\lambda-h\left(x, y ; \omega_{0}\right)}^{y}\left(\omega_{0}\right)$, we have $\omega_{0} \in \Omega_{y}(z)=\Omega_{x}(z) \subset \Omega_{x}(v)$, which shows that the $x$-open sets are $y$-open, and the first statement follows.

(ii) With $x, y, z$ and $\omega_{0}$ as above, since $\Omega_{x}(z)=\Omega_{y}(z)$, and $\nu_{x}\left(\Omega_{x}(z)\right)=N_{\lambda}^{-1}$, and $\nu_{y}\left(\Omega_{y}(z)\right)=N_{\lambda-h\left(x, y ; \omega_{0}\right)}^{-1}$, we see that

$$
\nu_{y}\left(\Omega_{x}(z)\right)=\nu_{y}\left(\Omega_{y}(z)\right)=N_{\lambda-h\left(x, y ; \omega_{0}\right)}^{-1}=N_{\lambda-h\left(x, y ; \omega_{0}\right)}^{-1} N_{\lambda} \nu_{x}\left(\Omega_{x}(z)\right),
$$

and so the measures are mutually absolutely continuous, and the Radon-Nikodym derivative is given by

$$
\frac{d \nu_{y}}{d \nu_{x}}(\omega)=\frac{N_{\lambda}}{N_{\lambda-h(x, y ; \omega)}} \quad \text { for any } \lambda \in P^{+} \text {such that } \lambda \gg \nu+\nu^{*} .
$$

The result follows from Proposition 3.15 by choosing $\lambda$ perhaps larger still so that both $\lambda$ and $\lambda-h(x, y ; \omega)$ are strongly dominant.

Let $r \in \operatorname{Hom}\left(P, \mathbb{C}^{\times}\right)$be the map

$$
\mu \mapsto \prod_{i=1}^{n} q_{t_{\lambda_{i}}}^{\frac{1}{2}\left\langle\mu, \alpha_{i}\right\rangle}=\prod_{\alpha \in R^{+}} \tau_{\alpha}^{\frac{1}{2}\langle\mu, \alpha\rangle}
$$

Following our usual convention we write $r^{\mu}$ in place of $r(\mu)$.

Proposition 3.15 immediately gives the following. 
Corollary 3.18. Let $\mu \in P$ be fixed. Then

$$
r^{\mu}=\left(\frac{N_{\lambda}}{N_{\lambda-\mu}}\right)^{1 / 2}
$$

for any $\lambda \in P^{++}$such that $\lambda-\mu \in P^{++}$.

For $\lambda \in P^{+}$, let us write $\mu \ggg \lambda$ to mean that $\mu-\Pi_{\lambda} \subset P^{++}$(in particular, notice that if $\mu \ggg \lambda$, then $\mu \gg \lambda$ and $\mu \in P^{++}$). The reason for introducing this notation is that we will want to ensure that the formula in Corollary 3.18 is applicable in the following results.

Recall the definition of the numbers $a_{\lambda, \mu ; \nu}$ from before (1.7).

Lemma 3.19. Let $\lambda \in P^{+}$. For each $x \in V_{P}, \omega \in \Omega, \mu \in \Pi_{\lambda}$ and $\nu \ggg \lambda$,

$$
\frac{1}{N_{\lambda}}\left|\left\{y \in V_{\lambda}(x) \mid h(x, y ; \omega)=\mu\right\}\right|=r^{-2 \mu} a_{\lambda, \nu-\mu ; \nu} .
$$

In particular, the value of the left hand side is independent of $x \in V_{P}$ and $\omega \in \Omega$.

Proof. We will first show that whenever $\nu \gg \lambda$,

$$
\left\{y \in V_{\lambda}(x) \mid h(x, y ; \omega)=\mu\right\}=V_{\lambda}(x) \cap V_{(\nu-\mu)^{*}}\left(v_{\nu}^{x}(\omega)\right) .
$$

If $y \in V_{\lambda}(x) \cap V_{(\nu-\mu)^{*}}\left(v_{\nu}^{x}(\omega)\right)$, then by Theorem 3.6] $v_{\nu}^{x}(\omega) \in \mathcal{S}^{y}(\omega) \cap V_{\nu-\mu}(y)$, and so $v_{\nu}^{x}(\omega)=v_{\nu-\mu}^{y}(\omega)$. Thus $h(x, y ; \omega)=\mu$.

Conversely, if $y \in V_{\lambda}(x)$ and $h(x, y ; \omega)=\mu$, then $v_{\nu}^{x}(\omega)=v_{\nu-\mu}^{y}(\omega)$ once $\nu \gg \lambda$ by Theorem 3.6. Thus $y \in V_{\lambda}(x) \cap V_{(\nu-\mu)^{*}}\left(v_{\nu}^{x}(\omega)\right)$.

Now suppose that $\nu \ggg \lambda$. By (1.7), (3.6) and Corollary 3.18 we have

$$
\frac{1}{N_{\lambda}}\left|\left\{y \in V_{\lambda}(x) \mid h(x, y ; \omega)=\mu\right\}\right|=\frac{N_{\nu-\mu}}{N_{\nu}} a_{\lambda, \nu-\mu ; \nu}=r^{-2 \mu} a_{\lambda, \nu-\mu ; \nu} .
$$

We now describe the algebra homomorphisms $h: \mathscr{A} \rightarrow \mathbb{C}$ in terms of (zonal) spherical functions.

Definition 3.20. Fix a vertex $x \in V_{P}$. A function $f: V_{P} \rightarrow \mathbb{C}$ is called spherical with respect to $x$ if

(i) $f(x)=1$,

(ii) $f$ is $x$-radial (that is, $f(y)=f\left(y^{\prime}\right)$ whenever $y, y^{\prime} \in V_{\lambda}(x)$ ), and

(iii) for each $A \in \mathscr{A}$ there is a number $c_{A}$ such that $A f=c_{A} f$.

The following is proved in $\left[8\right.$. Proposition 3.4] in the $\widetilde{A}_{2}$ case, and the proof there generalises immediately.

Proposition 3.21. An x-radial function $f: V_{P} \rightarrow \mathbb{C}$ is spherical if and only if the map $h: \mathscr{A} \rightarrow \mathbb{C}$ given by $h(A)=(A f)(x)$ defines an algebra homomorphism. Moreover, each $h \in \operatorname{Hom}(\mathscr{A}, \mathbb{C})$ arises in this way.

Let $x \in V_{P}$ be fixed and let $u \in \operatorname{Hom}\left(P, \mathbb{C}^{\times}\right)$and $y \in V_{P}$. Define

$$
F_{u}^{x}(y)=\int_{\Omega}(u r)^{h(x, y ; \omega)} d \nu_{x}(\omega)
$$

where $(u r)^{\lambda}=u^{\lambda} r^{\lambda}$ for all $\lambda \in P$. The integral exists by Theorem 3.4(ii) and the fact that $N_{\lambda}$ and $\left|\Pi_{\lambda}\right|$ are finite for each $\lambda \in P^{+}$.

In the following theorem we provide a second formula $h_{u}^{\prime}, u \in \operatorname{Hom}\left(P, \mathbb{C}^{\times}\right)$, for the algebra homomorphisms $\mathscr{A} \rightarrow \mathbb{C}$. In Theorem 6.2 we will show that $h_{u}^{\prime}=h_{u}$. 
Theorem 3.22. Let $x, y \in V_{P}$ with $y \in V_{\lambda}(x)$. Then for all $u \in \operatorname{Hom}\left(P, \mathbb{C}^{\times}\right)$

(i) $F_{u}^{x}(x)=1$,

(ii) $F_{u}^{x}(y)=F_{u}^{x^{\prime}}\left(y^{\prime}\right)$ whenever $x^{\prime}, y^{\prime} \in V_{P}$ satisfy $y^{\prime} \in V_{\lambda}\left(x^{\prime}\right)$, and

(iii) $A_{\lambda} F_{u}^{x}=\varphi_{\lambda}(u) F_{u}^{x}$, where for any $\nu \ggg \lambda$

$$
\varphi_{\lambda}(u)=\sum_{\mu \in \Pi_{\lambda}} r^{-\mu} a_{\lambda, \nu-\mu ; \nu} u^{\mu}
$$

which is independent of $x \in V_{P}$.

Thus the map $h_{u}^{\prime}: \mathscr{A} \rightarrow \mathbb{C}$ given by $h_{u}^{\prime}(A)=\left(A F_{u}^{x}\right)(x)$ defines an algebra homomorphism (by Proposition [3.21).

Proof. Since $\nu_{x}$ is a probability measure, (i) follows from Proposition 3.5

We now prove (ii), which we note is stronger than the claim that $F_{u}^{x}$ is $x$-radial. Let $\nu \in P^{+}$. Since $\Omega$ is the union of the disjoint sets $\Omega_{x}(z)$ over $z \in V_{\nu}(x)$, we have

$$
\begin{aligned}
F_{u}^{x}(y) & =\sum_{z \in V_{\nu}(x)} \int_{\Omega_{x}(z)}(u r)^{h(x, y ; \omega)} d \nu_{x}(\omega) \\
& =\sum_{\mu \in P^{+}} \sum_{z \in V_{\nu}(x) \cap V_{\mu}(y)} \int_{\Omega_{x}(z)}(u r)^{h(x, y ; \omega)} d \nu_{x}(\omega) .
\end{aligned}
$$

Now take $\nu \gg \lambda$, and so by Lemma $3.13 h(x, y ; \omega)=\nu-\mu$ for all $\omega \in \Omega_{x}(z)$ and $z \in V_{\nu}(x) \cap V_{\mu}(y)$. Since $\nu_{x}\left(\Omega_{x}(z)\right)=N_{\nu}^{-1}$ we have

$$
F_{u}^{x}(y)=\sum_{\mu \in P^{+}} \frac{1}{N_{\nu}}\left|V_{\nu}(x) \cap V_{\mu}(y)\right|(u r)^{\nu-\mu}
$$

and the result follows from (1.7).

We now prove (iii). Let $\nu \ggg \lambda$. By the cocycle relations (Proposition 3.5), Theorem 3.4(ii) and Lemma 3.19 we have

$$
\begin{aligned}
\left(A_{\lambda} F_{u}^{x}\right)(y) & =\frac{1}{N_{\lambda}} \sum_{z \in V_{\lambda}(y)} \int_{\Omega}(u r)^{h(x, z ; \omega)} d \nu_{x}(\omega) \\
& =\int_{\Omega}\left(\frac{1}{N_{\lambda}} \sum_{z \in V_{\lambda}(y)}(u r)^{h(y, z ; \omega)}\right)(u r)^{h(x, y ; \omega)} d \nu_{x}(\omega) \\
& =\left(\sum_{\mu \in \Pi_{\lambda}} r^{-\mu} a_{\lambda, \nu-\mu ; \nu} u^{\mu}\right) \int_{\Omega}(u r)^{h(x, y ; \omega)} d \nu_{x}(\omega) .
\end{aligned}
$$

Corollary 3.23. Let $y \in V_{\lambda}(x)$. Then for any $\omega \in \Omega$,

$$
h_{u}^{\prime}\left(A_{\lambda}\right)=F_{u}^{x}(y)=\frac{1}{N_{\lambda}} \sum_{z \in V_{\lambda}(x)}(u r)^{h(x, z ; \omega)}=\varphi_{\lambda}(u) .
$$

Proof. By Theorem 3.22(ii) and the definition of $A_{\lambda}$ we have $\left(A_{\lambda} F_{u}^{x}\right)(x)=F_{u}^{x}(y)$, and by Theorem 3.22 i) we have $\varphi_{\lambda}(u) F_{u}^{x}(x)=\varphi_{\lambda}(u)$. The result now follows from Theorem 3.22 (iii) and the proof thereof. 


\section{The Plancherel Measure}

Each $A \in \mathscr{A}$ maps $\ell^{2}\left(V_{P}\right)$ into itself, and for $\lambda \in P^{+}$and $f \in \ell^{2}\left(V_{P}\right)$ we have $\left\|A_{\lambda} f\right\|_{2} \leq\|f\|_{2}$ (see [7. Lemma 4.1] for a proof in a similar context). So we may regard $\mathscr{A}$ as a subalgebra of the $C^{*}$-algebra $\mathscr{L}\left(\ell^{2}\left(V_{P}\right)\right)$ of bounded linear operators on $\ell^{2}\left(V_{P}\right)$. The facts that $y \in V_{\lambda}(x)$ if and only if $x \in V_{\lambda^{*}}(y)$, and $N_{\lambda^{*}}=N_{\lambda}$, imply that $A_{\lambda}^{*}=A_{\lambda^{*}}$, and so the adjoint $A^{*}$ of any $A \in \mathscr{A}$ is also in $\mathscr{A}$.

Let $\mathscr{A}_{2}$ denote the completion of $\mathscr{A}$ with respect to $\|\cdot\|$, the $\ell^{2}$-operator norm. So $\mathscr{A}_{2}$ is a commutative $C^{*}$-algebra. We write $M_{2}=\operatorname{Hom}\left(\mathscr{A}_{2}, \mathbb{C}\right)$ (this is the maximal ideal space of $\left.\mathscr{A}_{2}\right)$, and we denote the associated Gelfand transform $\mathscr{A}_{2} \rightarrow \mathscr{C}\left(M_{2}\right)$ by $A \mapsto \hat{A}$, where $\hat{A}(h)=h(A)$. Here $\mathscr{C}\left(M_{2}\right)$ is the algebra of $\mathrm{w}^{*}$-continuous functions on $M_{2}$ with the sup norm. This map is an isometric isomorphism of $C^{*}$-algebras 9] Theorem I.3.1].

The algebra homomorphisms $\tilde{h}: \mathscr{A}_{2} \rightarrow \mathbb{C}$ are precisely the extensions to $\mathscr{A}_{2}$ of algebra homomorphisms $h: \mathscr{A} \rightarrow \mathbb{C}$ which are continuous with respect to the $\ell^{2}$-operator norm. When there is no risk of ambiguity we will simply write $h$ in place of $\tilde{h}$. If $h=h_{u}: \mathscr{A}_{2} \rightarrow \mathbb{C}$ we write $\hat{A}(u)$ in place of $\hat{A}(h)\left(\right.$ so $\widehat{A}_{\lambda}(u)=P_{\lambda}(u)$ ).

Let $\langle\cdot, \cdot\rangle$ be the usual inner product on $\ell^{2}\left(V_{P}\right)$ (this is not to be confused with the unrelated inner product on $E$ ). If $X \subset V_{P}$ we write $1_{X}$ for the characteristic function on $X$, and we write $\delta_{x}$ for $1_{\{x\}}$.

Lemma 4.1. Let $A \in \mathscr{A}_{2}$ and $o \in V_{P}$. Then $A \delta_{o}=0$ implies that $A=0$.

Proof. Let $x \in V_{P}$. Observe that if $A \in \mathscr{A}$ then $A \delta_{x}$ is $x$-radial, for if $A=$ $\sum_{\lambda} a_{\lambda} A_{\lambda}$ is a finite linear combination, then $A \delta_{x}=\sum_{\lambda} a_{\lambda} N_{\lambda}^{-1} 1_{V_{\lambda}(x)}$, which is $x$-radial. It follows that $A \delta_{x}$ is $x$-radial for all $A \in \mathscr{A}_{2}$. Now, given $A \in \mathscr{A}_{2}$ and $\mu \in P^{+},\left\langle A \delta_{x}, 1_{V_{\mu^{*}(x)}}\right\rangle$ does not depend on $x \in V_{P}$, for if $A=\sum_{\lambda} a_{\lambda} A_{\lambda} \in \mathscr{A}$, then $\left\langle A \delta_{x}, 1_{V_{\mu^{*}}(x)}\right\rangle=a_{\mu}$. Thus if $A \in \mathscr{A}_{2}$ and $A \delta_{o}=0$, then $\left\langle A \delta_{o}, 1_{V_{\mu^{*}}(o)}\right\rangle=0$ for all $\mu \in P^{+}$, and so $\left\langle A \delta_{x}, 1_{V_{\mu^{*}}(x)}\right\rangle=0$ for all $\mu \in P^{+}$and for all $x \in V_{P}$. Since $A \delta_{x}$ is $x$-radial for all $x \in V_{P}$, it follows that $A \delta_{x}=0$ for all $x$, and so $A f=0$ for all finitely supported functions $f \in \ell^{2}\left(V_{P}\right)$. Thus by density the same is true for all $f \in \ell^{2}\left(V_{P}\right)$, completing the proof.

Since $A_{\lambda} \delta_{o}=N_{\lambda}^{-1} 1_{V_{\lambda^{*}}(o)}$ for each $o \in V_{P}$, we have $\left\langle A_{\lambda} \delta_{o}, A_{\mu} \delta_{o}\right\rangle=\delta_{\lambda, \mu} N_{\lambda}^{-1}$. Thus by Lemma 4.1 we can define an inner product on $\mathscr{A}_{2}$ (independent of $o \in V_{P}$ ) by $\langle A, B\rangle=\left\langle A \delta_{o}, B \delta_{o}\right\rangle$.

For any fixed $o \in V_{P}$, the map $A \mapsto\left\langle A, A_{0}\right\rangle=\left(A \delta_{o}\right)(o)$ maps the identity $A_{0}$ of $\mathscr{A}_{2}$ to 1 and satisfies $\left|\left\langle A, A_{0}\right\rangle\right| \leq\left\|A \delta_{o}\right\|_{2} \leq\|A\|=\|\hat{A}\|_{\infty}$. Thus by the Riesz Representation Theorem there exists a unique regular Borel probability measure $\pi$ on $M_{2}$ so that

$$
\left(A \delta_{o}\right)(o)=\int_{M_{2}} \hat{A}(h) d \pi(h) \text { for all } A \in \mathscr{A}_{2} .
$$

Hence, for all $A, B \in \mathscr{A}_{2}$,

$$
\langle A, B\rangle=\left\langle A \delta_{o}, B \delta_{o}\right\rangle=\left(B^{*} A \delta_{o}\right)(o)=\int_{M_{2}} \hat{A}(h) \overline{\hat{B}(h)} d \pi(h) .
$$

We refer to $\pi$ and $M_{2}$ as the Plancherel measure and spectrum of $\mathscr{A}_{2}$, respectively.

Proposition 4.2. $M_{2}=\operatorname{supp}(\pi)$. 
Proof. If $h_{0} \in M_{2} \backslash \operatorname{supp}(\pi)$, then by Urysohn's Lemma there is a $\varphi \in \mathscr{C}\left(M_{2}\right)$ so that $\varphi=0$ on $\operatorname{supp}(\pi)$ and $\varphi\left(h_{0}\right)=1$. Since $A \mapsto \hat{A}$ is an isomorphism, there is an $A \in \mathscr{A}_{2}$ so that $\hat{A}=\varphi$. Then by (4.1)

$$
\left\|A \delta_{o}\right\|_{2}^{2}=\langle A, A\rangle=\int_{\operatorname{supp}(\pi)}|\hat{A}(h)|^{2} d \pi(h)=0,
$$

and so $A=0$ by Lemma 4.1 contradicting $\hat{A}=\varphi \neq 0$.

\section{Calculating the Plancherel Measure and the $\ell^{2}$-spectrum}

In this section we will calculate the Plancherel measure of $\mathscr{A}_{2}$. It turns out that there are two cases to consider. We will then use these results to compute the $\ell^{2}$-spectrum of $\mathscr{A}$. The Plancherel measure will also be used in the proof of Theorem 6.2 where we show that $h_{u}=h_{u}^{\prime}$ for all $u \in \operatorname{Hom}\left(P, \mathbb{C}^{\times}\right)$.

Lemma 5.1. $\tau_{\alpha}<1$ for some $\alpha \in R$ if and only if $R=B C_{n}$ and $q_{n}<q_{0}$.

Proof. If $R$ is reduced we have $\tau_{\alpha}=q_{\alpha}$ for all $\alpha \in R$. Thus $\tau_{\alpha}<1$ for some $\alpha \in R$ implies that $R=B C_{n}$ for some $n \geq 1$. Thus $R$ may be described as follows (see [2] VI, $\S 4$, No.14]). Let $E=\mathbb{R}^{n}$ with standard basis $\left\{e_{i}\right\}_{i=1}^{n}$, and let $R$ consist of the vectors $\pm e_{i}, \pm 2 e_{i}$ and $\pm e_{j} \pm e_{k}$ for $1 \leq i \leq n$ and $1 \leq j<k \leq n$. Recall from 15. Appendix] that in an affine building of type $B C_{n}$ we have $q_{1}=\cdots=q_{n-1}$. Thus by the definition of the numbers $\tau_{\alpha}$ we have $\tau_{ \pm e_{i}}=q_{n} q_{0}^{-1}, \tau_{ \pm 2 e_{i}}=q_{0}$ and $\tau_{ \pm e_{j} \pm e_{k}}=q_{1}$ for $1 \leq i \leq n$ and $1 \leq j<k \leq n$. The result follows.

Following [12, Chapter V] we call the situation where $\tau_{\alpha} \geq 1$ for all $\alpha \in R$ the standard case, and we call the situation where $\tau_{\alpha}<1$ for some $\alpha \in R$ the exceptional case.

5.1. The Standard Case. Let $\mathbb{U}=\left\{u \in \operatorname{Hom}\left(P, \mathbb{C}^{\times}\right):\left|u^{\lambda}\right|=1\right.$ for all $\left.\lambda \in P\right\}$. Writing $u_{i}=u^{\lambda_{i}}$ for each $i=1, \ldots, n$, we have $\mathbb{U} \cong \mathbb{T}^{n}$ where $\mathbb{T}=\{z \in \mathbb{C}:|z|=1\}$.

In the next theorem we introduce (following [12]) a measure $\pi_{0}$ which we will shortly see is closely related to the Plancherel measure $\pi$ (in the standard case). We will write $\hat{A}(u)$ for $h_{u}(A)$ when $A \in \mathscr{A}$ and $u \in \mathbb{U}$. As we shall see in Corollary [5.4] each such $h_{u}$ is continuous for the $\ell^{2}$-operator norm, and so (5.1) will also be valid for $A, B \in \mathscr{A}_{2}$.

Theorem 5.2. ( $c f$. 12, Theorem 5.1.5]) Let du denote the normalised Haar measure on $\mathbb{U}$, and let $\pi_{0}$ be the measure on $\mathbb{U}$ given by $d \pi_{0}(u)=\frac{W_{0}\left(q^{-1}\right)}{\left|W_{0}\right|}|c(u)|^{-2} d u$. Then

$$
\langle A, B\rangle=\int_{\mathbb{U}} \hat{A}(u) \overline{\hat{B}(u)} d \pi_{0}(u) \quad \text { for all } A, B \in \mathscr{A} .
$$

Proof. We may assume that $A=A_{\mu}$ and $B=A_{\nu}$, where $\mu, \nu \in P^{+}$. Then the integrand in (5.1) is $\widehat{A_{\mu} A_{\nu^{*}}}(u)$. Now $A_{\mu} A_{\nu^{*}}=\sum_{\lambda \in P^{+}} a_{\mu, \nu^{*} ; \lambda} A_{\lambda}$, and since $a_{\mu, \nu^{*} ; \lambda}=\delta_{\mu, \nu} / N_{\mu}$, it suffices to show that $\int_{\mathbb{U}} \widehat{A}_{\lambda}(u) d \pi_{0}(u)=\delta_{\lambda, 0}$ for each $\lambda \in P^{+}$. Notice that if $u \in \mathbb{U}$, then $\bar{u}=u^{-1}$, and so

$$
|c(u)|^{2}=c(u) c\left(u^{-1}\right)=\prod_{\alpha \in R} \frac{1-\tau_{\alpha}^{-1} \tau_{\alpha / 2}^{-1 / 2} u^{-\alpha^{\vee}}}{1-\tau_{\alpha / 2}^{-1 / 2} u^{-\alpha^{\vee}}} .
$$


Thus $|c(w u)|^{2}=|c(u)|^{2}$ for all $w \in W_{0}$. Furthermore, if $f(u)=\sum_{\lambda} a_{\lambda} u^{\lambda}$ is such that $\sum_{\lambda}\left|a_{\lambda}\right|<\infty$, then $\int_{\mathbb{U}} f(u) d u=a_{0}$. It follows that $\int_{\mathbb{U}} f(w u) d u=\int_{\mathbb{U}} f(u) d u$ for all $w \in W_{0}$.

Using these facts we see that

$$
\int_{\mathbb{U}} \widehat{A}_{\lambda}(u) d \pi_{0}(u)=q_{t_{\lambda}}^{-1 / 2} \int_{\mathbb{U}} \frac{u^{\lambda}}{c\left(u^{-1}\right)} d u .
$$

Let $R_{\tau}^{+}=\left\{\alpha \in R^{+} \mid \tau_{\alpha} \neq 1\right\}$. Then it is clear that we can write

$$
\frac{1}{c\left(u^{-1}\right)}=\prod_{\alpha \in R_{\tau}^{+}} \frac{1-\tau_{\alpha / 2}^{-1 / 2} u^{\alpha^{\vee}}}{1-\tau_{\alpha}^{-1} \tau_{\alpha / 2}^{-1 / 2} u^{\alpha^{\vee}}}=\sum_{\gamma \in Q^{+}} a_{\gamma} u^{\gamma}
$$

where $a_{0}=1$ and the series is uniformly convergent. Since $\left\{\lambda_{i}\right\}_{i=1}^{n}$ forms an acute basis of $E$ [2, VI, $\S 1$, No.10] we have $\left\langle\lambda, \lambda_{i}\right\rangle \geq 0$ for all $\lambda \in P^{+}$and for all $1 \leq i \leq n$. Thus each $\lambda \in P^{+}$is a linear combination of $\left\{\alpha_{i}\right\}_{i=1}^{n}$ with nonnegative coefficients. It follows that if $\lambda \in P^{+}, \gamma \in Q^{+}$and $\lambda+\gamma=0$, then $\lambda=\gamma=0$. Hence by (5.2) we have $\int_{\mathbb{U}} \widehat{A}_{\lambda}(u) d \pi_{0}(u)=\delta_{\lambda, 0}$, completing the proof.

Fix $o \in V_{P}$ and let $\ell_{o}^{2}\left(V_{P}\right)$ denote the space of all $f \in \ell^{2}\left(V_{P}\right)$ which are constant on each set $V_{\lambda}(o)$. For $A \in \mathscr{A}_{2}$ define $\|A\|_{o}$ by

$$
\|A\|_{o}=\sup \left\{\|A f\|_{2}: f \in \ell_{o}^{2}\left(V_{P}\right) \text { and }\|f\|_{2} \leq 1\right\},
$$

which defines a norm on $\mathscr{A}_{2}$ (see Lemma 4.1), and clearly $\|A\|_{o} \leq\|A\|$ for all $A \in \mathscr{A}_{2}$.

Remark 5.3. In fact $\|A\|_{o}=\|A\|$ for all $A \in \mathscr{A}_{2}$ (in both the standard and exceptional cases). To see this, recall that an injective homomorphism between two $C^{*}$-algebras is an isometry [9] Theorem I.5.5]. Let $\Phi: \mathscr{A}_{2} \rightarrow \mathscr{L}\left(\ell_{o}^{2}\left(V_{P}\right)\right)$ be the linear map given by $\left.A \mapsto A\right|_{\ell_{o}^{2}\left(V_{P}\right)}$. Since $\|A\|_{o}=\left\|\left.A\right|_{\ell_{o}^{2}\left(V_{P}\right)}\right\|$, it suffices to show that $\Phi$ is an injection. This is clear from Lemma 4.1 for $\Phi(A)=0$ implies that $A \delta_{o}=0$, and so $A=0$.

Corollary 5.4. Each $h_{u}, u \in \mathbb{U}$, is continuous for the $\ell^{2}$-operator norm.

Proof. We show that $\left|h_{u}(A)\right| \leq\|A\|_{o}$ for all $A \in \mathscr{A}$ and $u \in \mathbb{U}$. Suppose that this condition fails for some $u_{0} \in \mathbb{U}$ and $A \in \mathscr{A}$. Then there exists $\delta>0$ so that $\left|h_{u_{0}}(A)\right|>(1+\delta)\|A\|_{o}$. Since $h_{u}(A)$ is a Laurent polynomial in $u_{1}, \ldots, u_{n}$ there exists a neighbourhood $\mathcal{N}$ of $u_{0}$ in $\mathbb{U}$ such that $\left|h_{u}(A)\right|>(1+\delta)\|A\|_{o}$ for all $u \in \mathcal{N}$. Let $\mathcal{N}^{\prime}$ denote the set of $u \in \mathbb{U}$ such that $\left|h_{u}(A)\right|>(1+\delta)\|A\|_{o}$, so $W_{0} \mathcal{N}^{\prime}=\mathcal{N}^{\prime}$. Let $\mathbb{U} / W_{0}$ denote the set of $W_{0}$ orbits in $\mathbb{U}$. It is compact Hausdorff with respect to the quotient topology, and

$$
\mathscr{C}\left(\mathbb{U} / W_{0}\right) \cong\left\{\varphi \in \mathscr{C}(\mathbb{U}) \mid \varphi(w u)=\varphi(u) \text { for all } w \in W_{0} \text { and } u \in \mathbb{U}\right\} .
$$

Now there exists $\varphi \in \mathscr{C}\left(\mathbb{U} / W_{0}\right)$ not identically 0 which is 0 outside $\mathcal{N}^{\prime}$. By the Stone-Weierstrass Theorem, for any given $\epsilon>0$ there exists $B \in \mathscr{A}$ so that $\| \hat{B}-$ $\varphi \|_{\infty}<\epsilon$, and choosing $\epsilon$ suitably small we can ensure that

$$
\int_{\mathcal{N}^{\prime}}|\hat{B}(u)|^{2} d \pi_{0}(u) \geq \frac{1}{1+\delta} \int_{\mathbb{U}}|\hat{B}(u)|^{2} d \pi_{0}(u)>0 .
$$


Thus by (5.1)

$$
\begin{aligned}
\left\|A B \delta_{o}\right\|_{2}^{2} & =\langle A B, A B\rangle \\
& =\int_{\mathbb{U}}|\hat{A}(u)|^{2}|\hat{B}(u)|^{2} d \pi_{0}(u) \\
& \geq(1+\delta)^{2}\|A\|_{o}^{2} \int_{\mathcal{N}^{\prime}}|\hat{B}(u)|^{2} d \pi_{0}(u) \\
& \geq(1+\delta)\|A\|_{o}^{2}\left\|B \delta_{o}\right\|_{2}^{2},
\end{aligned}
$$

and so $\|A f\|_{2} \geq \sqrt{1+\delta}\|A\|_{o}\|f\|_{2}$ for $f=B \delta_{o}$, contrary to the definition of $\|A\|_{o}$.

Corollary 5.5. In the standard case, $M_{2}=\left\{\tilde{h}_{u} \mid u \in \mathbb{U}\right\}$. Moreover, the map $\varpi: u \mapsto \tilde{h}_{u}$ induces a homeomorphism $\mathbb{U} / W_{0} \rightarrow M_{2}$ (where $\mathbb{U}$ is given the Euclidean topology, $\mathbb{U} / W_{0}$ is given the quotient topology, and $M_{2}$ is given the $w^{*}$-topology), and the Plancherel measure $\pi$ is the image of the measure $\pi_{0}$ of Theorem 5.2 under $\varpi$.

Proof. The w*-topology on $M_{2}$, defined using the functionals $h \mapsto h(A), A \in \mathscr{A}_{2}$, is compact, and so agrees with the topology defined using only the functionals $h \mapsto h(A), A \in \mathscr{A}$, since the latter is Hausdorff. Since each $h_{u}(A)(A \in \mathscr{A}$ fixed) is a Laurent polynomial in $u_{1}, \ldots, u_{n}$, the map $\varpi: u \mapsto \tilde{h}_{u}$, defined from $\mathbb{U}$ to $M_{2}$ in light of Corollary [5.4 is continuous. Thus $\varpi(\mathbb{U})$ is closed in $M_{2}$, and $\varpi$ induces a homeomorphism $\mathbb{U} / W_{0} \rightarrow \varpi(\mathbb{U})$ since $\mathbb{U} / W_{0}$ is compact. The image $\pi$ of $\pi_{0}$ under $\varpi$ satisfies the defining properties of the Plancherel measure. Since $M_{2}=\operatorname{supp}(\pi)$ by Proposition 4.2

$$
\pi\left(M_{2} \backslash \varpi(\mathbb{U})\right)=\pi_{0}\left(\varpi^{-1}\left(M_{2} \backslash \varpi(\mathbb{U})\right)\right)=0,
$$

and so $M_{2}=\varpi(\mathbb{U})$. Thus $\varpi$ is surjective, and it is injective by Proposition 2.1

5.2. The Exceptional Case. Let $R=B C_{n}$ for some $n \geq 1$ and suppose that $q_{n}<q_{0}$. Recall the description of $R$ from the proof of Lemma 5.1] Let $\alpha_{i}=e_{i}-e_{i+1}$ for $1 \leq i \leq n-1$ and let $\alpha_{n}=e_{n}$. The set $B=\left\{\alpha_{i}\right\}_{i=1}^{n}$ is a base of $R$, and the set of positive roots with respect to $B$ is

$$
R^{+}=\left\{e_{i}, 2 e_{i}, e_{j}-e_{k}, e_{j}+e_{k} \mid 1 \leq i \leq n, 1 \leq j<k \leq n\right\} .
$$

Recall (from the proof of Lemma 5.1) that $q_{1}=\cdots=q_{n-1}$ in this case. Let $a=\sqrt{q_{n} q_{0}}$ and $b=\sqrt{q_{n} / q_{0}}$ (so $\left.b<1\right)$.

Let $u \in \operatorname{Hom}\left(P, \mathbb{C}^{\times}\right)$. Since $e_{i} \in P$ for each $i=1, \ldots, n$, we may define numbers $t_{i}=t_{i}(u)$ by $t_{i}=u^{e_{i}}$. We will now give a formula for $c(u)$ in this case in terms of the numbers $\left\{t_{i}\right\}_{i=1}^{n}$ (see Remark 2.2 for a related discussion).

If $\alpha=2 e_{i}, 1 \leq i \leq n$, then $\alpha \in R_{1} \backslash R_{3}$, and so $\tau_{\alpha}=q_{0}$. Now $\alpha / 2=e_{i} \in R_{2} \backslash R_{3}$, and so $\tau_{\alpha / 2}=q_{\alpha / 2} q_{0}^{-1}$. Since $|\alpha / 2|=\left|\alpha_{n}\right|$ we have $q_{\alpha / 2}=q_{\alpha_{n}}=q_{n}$, and thus $\tau_{\alpha / 2}=q_{n} q_{0}^{-1}$. Now if $\alpha=e_{i}, 1 \leq i \leq n$, then $\alpha \in R_{2} \backslash R_{3}$, and so by the above $\tau_{\alpha}=q_{n} q_{0}^{-1}$, and since $\alpha / 2 \notin R$ we have $\tau_{\alpha / 2}=1$. Since $\left(2 e_{i}\right)^{\vee}=e_{i}$ and $e_{i}^{\vee}=2 e_{i}$, the factors in $c(u)$ (see (2.1) ) corresponding to the roots $\alpha=2 e_{i}$ and $\alpha=e_{i}$ are

$$
\frac{1-q_{n}^{-1 / 2} q_{0}^{-1 / 2} t_{i}^{-1}}{1-q_{n}^{-1 / 2} q_{0}^{1 / 2} t_{i}^{-1}} \cdot \frac{1-q_{n}^{-1} q_{0} t_{i}^{-2}}{1-t_{i}^{-2}}=\frac{\left(1-a^{-1} t_{i}^{-1}\right)\left(1+b^{-1} t_{i}^{-1}\right)}{1-t_{i}^{-2}} .
$$

If $\alpha=e_{j} \pm e_{k}, 1 \leq j<k \leq n$, then $\alpha \in R_{3}$, and so $\tau_{\alpha}=q_{\alpha}$. Since $|\alpha|=$ $\left|e_{1}-e_{2}\right|=\left|\alpha_{1}\right|$ we have $q_{\alpha}=q_{\alpha_{1}}=q_{1}\left(=q_{2}=\cdots=q_{n-1}\right)$, and so the product 
of the two factors of $c(u)$ corresponding to the roots $\alpha=e_{j}-e_{k}$ and $\alpha=e_{j}+e_{k}$ $(1 \leq j<k \leq n)$ is

$$
\frac{\left(1-q_{1}^{-1} t_{j}^{-1} t_{k}\right)}{\left(1-t_{j}^{-1} t_{k}\right)} \cdot \frac{\left(1-q_{1}^{-1} t_{j}^{-1} t_{k}^{-1}\right)}{\left(1-t_{j}^{-1} t_{k}^{-1}\right)} .
$$

Combining all these factors we see that $c(u)$ equals

$$
\left\{\prod_{i=1}^{n} \frac{\left(1-a^{-1} t_{i}^{-1}\right)\left(1+b^{-1} t_{i}^{-1}\right)}{1-t_{i}^{-2}}\right\}\left\{\prod_{1 \leq j<k \leq n} \frac{\left(1-q_{1}^{-1} t_{j}^{-1} t_{k}\right)\left(1-q_{1}^{-1} t_{j}^{-1} t_{k}^{-1}\right)}{\left(1-t_{j}^{-1} t_{k}\right)\left(1-t_{j}^{-1} t_{k}^{-1}\right)}\right\} .
$$

Notice that when $R=B C_{n}, Q=P$, and so we will be able to apply the results of 12] (see the paragraph after the proof of Proposition 2.11). Thus when $q_{1}>1$ the Plancherel measure here depends on how many of the numbers $q_{1}^{k} b, k \in \mathbb{N}$, are less than 1 (see [12, page 70]). Since we have an underlying building we have the following simplification.

Lemma 5.6. If $q_{1}>1$, then $q_{1} b \geq 1$.

Proof. By a well known theorem of D. Higman (see [17, page 30] for example), in a finite thick generalised 4-gon with parameters $(k, l)$, we have $k \leq l^{2}$ and $l \leq k^{2}$. Thus by [17, Theorem 3.5 and Proposition 3.2] we have $q_{1}^{2} \geq q_{0}$ (even if $q_{0}=1$ ), and so $q_{1} b \geq \sqrt{q_{n}} \geq 1$.

Let $\mathbb{T}=\{z \in \mathbb{C}:|z|=1\}$. Let $d t=d t_{1} \cdots d t_{n}$, where $d t_{i}$ is normalised Haar measure on $\mathbb{T}$. Define $\phi_{0}(u)=c(u) c\left(u^{-1}\right)$ and

$$
\phi_{1}(u)=\lim _{t_{1} \rightarrow-b} \frac{\phi_{0}(u)}{1+b^{-1} t_{1}} \quad \text { and } \quad d t^{\prime}=d \delta_{-b}\left(t_{1}\right) d t_{2} \cdots d t_{n} .
$$

Note that this limit exists since there is a factor $1+b^{-1} t_{1}$ in $c\left(u^{-1}\right)$. We use the isomorphism $\mathbb{U} \rightarrow \mathbb{T}^{n}, u \mapsto\left(t_{1}, \ldots, t_{n}\right)$ to identify $\mathbb{U}$ with $\mathbb{T}^{n}$. Define $\mathbb{U}^{\prime}=$ $\{-b\} \times \mathbb{T}^{n-1}$, and write $U=\mathbb{U} \cup \mathbb{U}^{\prime}$.

Theorem 5.7. Let $\pi_{0}$ be the measure on $U=\mathbb{U} \cup \mathbb{U}^{\prime}$ given by $d \pi_{0}(u)=\frac{W_{0}\left(q^{-1}\right)}{\left|W_{0}\right|} \frac{d t}{\phi_{0}(u)}$ on $\mathbb{U}$ and $d \pi_{0}(u)=\frac{W_{0}\left(q^{-1}\right)}{\left|W_{0}^{\prime}\right|} \frac{d t^{\prime}}{\phi_{1}(u)}$ on $\mathbb{U}^{\prime}$, where $W_{0}^{\prime}$ is the Coxeter group $C_{n-1}$ (with $C_{1}=A_{1}$ and $\left.C_{0}=\{1\}\right)$. Then (in the exceptional case)

$$
\langle A, B\rangle=\int_{U} \hat{A}(u) \overline{\hat{B}(u)} d \pi_{0}(u) \quad \text { for all } A, B \in \mathscr{A} .
$$

Proof. When $q_{1}>1$ this follows from the 'group free' calculations made in 12 , Theorem 5.2.10], taking into account Lemma [5.6 If $q_{1}=1$ the formula for $c(u)$ simplifies considerably, and a calculation similar to that in [12, Theorem 5.2.10] proves the result in this case too.

As in the standard case we have the following corollary (see Corollary [5.5).

Corollary 5.8. In the exceptional case, $M_{2}=\left\{\tilde{h}_{u} \mid u \in U\right\}$. Moreover, the map $\varpi: u \mapsto \tilde{h}_{u}$ induces a homeomorphism $\left(\mathbb{U} / W_{0}\right) \cup\left(\mathbb{U}^{\prime} / W_{0}^{\prime}\right) \rightarrow M_{2}$ and the Plancherel measure $\pi$ is the image of the measure $\pi_{0}$ of Theorem 5.7 under $\varpi$. 


\section{EQUaLity OF $h_{u}$ AND $h_{u}^{\prime}$}

In this section we show that $h_{u}=h_{u}^{\prime}$ for all $u \in \operatorname{Hom}\left(P, \mathbb{C}^{\times}\right)$, where $h_{u}^{\prime}$ is as in Theorem 3.22 (see also Corollary [3.23). To conveniently state our results we will write $U=\mathbb{U}$ in the standard case. Thus $U=\mathbb{U}$ in the standard case and $U=\mathbb{U} \cup \mathbb{U}^{\prime}$ in the exceptional case.

Lemma 6.1. Let $\lambda, \mu, \nu \in P^{+}$. Then

(i) $\int_{U} P_{\lambda}(u) \overline{P_{\mu}(u)} d \pi_{0}(u)=\delta_{\lambda, \mu} N_{\lambda}^{-1}$, and

(ii) $a_{\lambda, \mu ; \nu}=N_{\nu} \int_{U} P_{\lambda}(u) P_{\mu}(u) \overline{P_{\nu}(u)} d \pi_{0}(u)$.

Proof. (i) Since each $h_{u}, u \in U$, is continuous with respect to the $\ell^{2}$-operator norm (see Corollary [5.5] and Corollary [5.8) we have $P_{\lambda}(u)=\widehat{A}_{\lambda}(u)$ for all $\lambda \in P^{+}$and all $u \in U$. Thus by (5.1) in the standard case, and (5.3) in the exceptional case,

$$
\int_{U} P_{\lambda}(u) \overline{P_{\mu}(u)} d \pi_{0}(u)=\left\langle A_{\lambda}, A_{\mu}\right\rangle=N_{\lambda}^{-1} \delta_{\lambda, \mu} .
$$

(ii) Using the previous part we have

$$
\int_{U} P_{\lambda}(u) P_{\mu}(u) \overline{P_{\nu}(u)} d \pi_{0}(u)=\sum_{\eta \in P^{+}}\left(a_{\lambda, \mu ; \eta} \int_{U} P_{\eta}(u) \overline{P_{\nu}(u)} d \pi_{0}(u)\right)=N_{\nu}^{-1} a_{\lambda, \mu ; \nu},
$$

completing the proof.

Theorem 6.2. $h_{u}^{\prime}=h_{u}$ for all $u \in \operatorname{Hom}\left(P, \mathbb{C}^{\times}\right)$.

Proof. From (B.1) we have

$$
h_{u}\left(A_{\lambda}\right)=P_{\lambda}(u)=\sum_{\mu \in \Pi_{\lambda}} a_{\lambda, \mu} u^{\mu}
$$

for some numbers $a_{\lambda, \mu}$. On the other hand, by Theorem 3.22 and Corollary 3.23 we have

$$
h_{u}^{\prime}\left(A_{\lambda}\right)=\sum_{\mu \in \Pi_{\lambda}} r^{-\mu} a_{\lambda, \nu-\mu ; \nu} u^{\mu} \quad \text { for any } \nu \ggg \lambda .
$$

We will show that for all $\mu \in \Pi_{\lambda}, a_{\lambda, \mu}=r^{-\mu} a_{\lambda, \nu-\mu ; \nu}$ provided that $\nu \ggg \lambda$. Comparing formulae (6.1) and (6.2) this evidently proves that $h_{u}=h_{u}^{\prime}$ for all $u \in \operatorname{Hom}\left(P, \mathbb{C}^{\times}\right)$.

Let us first consider the standard case, so $U=\mathbb{U}$. Let $\mu \in \Pi_{\lambda}$ and $\nu \ggg \lambda$. By Corollary 3.18 we have $r^{-\mu}=\sqrt{N_{\nu-\mu} / N_{\nu}}$, and so by (1.6) we compute $r^{-\mu} N_{\nu}=$ $W_{0}\left(q^{-1}\right) q_{t_{\nu}}^{1 / 2} q_{t_{\nu-\mu}}^{1 / 2}$. Thus by Lemma 6.1(ii),

$$
\begin{aligned}
r^{-\mu} a_{\lambda, \nu-\mu ; \nu} & =r^{-\mu} N_{\nu} \int_{\mathbb{U}} P_{\lambda}(u) P_{\nu-\mu}(u) \overline{P_{\nu}(u)} d \pi_{0}(u) \\
& =W_{0}\left(q^{-1}\right) q_{t_{\nu}}^{1 / 2} \int_{\mathbb{U}} P_{\lambda}(u) \overline{P_{\nu}(u)} \frac{u^{\nu-\mu}}{c\left(u^{-1}\right)} d u .
\end{aligned}
$$

Since $\left|c\left(w u^{-1}\right)\right|^{2}=\left|c\left(u^{-1}\right)\right|^{2}$ for all $u \in \mathbb{U}$ we see that $c\left(w u^{-1}\right) / c\left(u^{-1}\right) \in L^{1}(\mathbb{U})$ for all $w \in W_{0}$, and so by (6.3) we have

$$
r^{-\mu} a_{\lambda, \nu-\mu ; \nu}=\sum_{w \in W_{0}} \int_{\mathbb{U}} u^{\nu-w \nu}\left(P_{\lambda}(u) u^{-\mu} \frac{c\left(w u^{-1}\right)}{c\left(u^{-1}\right)}\right) d u
$$


We claim that the integral in (6.4) is 0 for all $w \neq 1$. To see this, notice that by Lemma 3.19 $r^{-\mu} a_{\lambda, \nu-\mu ; \nu}, \mu \in \Pi_{\lambda}$, is independent of $\nu \ggg \lambda$, and so we may choose $\nu=N\left(\lambda_{1}+\cdots+\lambda_{n}\right)$ for suitably large $N \in \mathbb{N}$. Suppose $w \neq 1$. Since $w\left(\lambda_{1}+\cdots+\lambda_{n}\right) \neq \lambda_{1}+\cdots+\lambda_{n}$ we see that

$$
\left|\left\langle\lambda_{1}+\cdots+\lambda_{n}-w\left(\lambda_{1}+\cdots+\lambda_{n}\right), \alpha_{i_{0}}\right\rangle\right| \geq 1
$$

for at least one $i_{0} \in I_{0}$, and so $\left|\left\langle\nu-w \nu, \alpha_{i_{0}}\right\rangle\right| \geq N$. Thus by the Riemann-Lebesgue Lemma $\lim _{N \rightarrow \infty} \int_{\mathbb{U}} u^{\nu-w \nu} f(u) d u=0$ for all $f \in L^{1}(\mathbb{U})$. Thus using (6.1) we have

$$
r^{-\mu} a_{\lambda, \nu-\mu ; \nu}=\int_{\mathbb{U}} P_{\lambda}(u) u^{-\mu} d u=a_{\lambda, \mu} .
$$

Let us now prove the result in the exceptional case, where $U=\mathbb{U} \cup \mathbb{U}^{\prime}$. Following the above we have

$$
r^{-\mu} a_{\lambda, \nu-\mu ; \nu}=a_{\lambda, \mu}+r^{-\mu} N_{\nu} \int_{\mathbb{U}^{\prime}} P_{\lambda}(u) P_{\nu-\mu}(u) \overline{P_{\nu}(u)} d \pi_{0}(u)
$$

whenever $\nu \ggg \lambda$. We will show that the integral in (6.5) is zero.

The group $W_{0}$ acts on $\left\{e_{i}\right\}_{i=1}^{n}$ as the group of all signed permutations. Since $t_{i}=u^{e_{i}}$, for each $w \in W_{0}$ we have $w\left(t_{1}, \ldots, t_{n}\right)=\left(t_{\sigma_{w}(1)}^{\epsilon_{w}(1)}, \ldots, t_{\sigma_{w}(n)}^{\epsilon_{w}(n)}\right)$ where $\sigma_{w}$ is a permutation of $\{1, \ldots, n\}$ and $\epsilon_{w}:\{1, \ldots, n\} \rightarrow\{1,-1\}$. By directly examining the formula for $c(u)$ we see that if $\epsilon_{w}\left(\sigma_{w}(1)\right)=-1$ then $\left.c(w u)\right|_{t_{1}=-b}=0$. Write $W_{0}^{+}=\left\{w \in W_{0} \mid \epsilon_{w}\left(\sigma_{w}(1)\right)=1\right\}$. Note that for all $\lambda \in P^{+}, \lambda^{*}=\lambda$. Thus $\overline{P_{\lambda}(u)}=P_{\lambda}(u)$ for all $u \in U$. Following the calculation in the standard case, and using the above observations, we see that

$$
\begin{aligned}
r^{-\mu} a_{\lambda, \nu-\mu ; \nu}-a_{\lambda, \mu} & =r^{-\mu} N_{\nu} \int_{\mathbb{U}^{\prime}} P_{\lambda}(u) P_{\nu-\mu}(u) P_{\nu}(u) d \pi_{0}(u) \\
& =\frac{1}{\left|W_{0}^{\prime}\right|} \sum_{w, w^{\prime} \in W_{0}^{+}} \int_{\mathbb{U}^{\prime}} u^{w \nu+w^{\prime} \nu}\left(P_{\lambda}(u) u^{-w \mu} \frac{c(w u) c\left(w^{\prime} u\right)}{\phi_{1}(u)}\right) d t^{\prime} .
\end{aligned}
$$

Since $w_{0}=-1$ it is clear that if $w \in W_{0}^{+}$then $w_{0} w \notin W_{0}^{+}$. Take any pair $w, w^{\prime} \in W_{0}^{+}$. As before, let $\nu=N\left(\lambda_{1}+\cdots+\lambda_{n}\right)$ for sufficiently large $N$. Since $w^{\prime} \neq w_{0} w$ we have $w \nu+w^{\prime} \nu \neq 0$. The same argument as in the standard case now shows that $\left|\left\langle w \nu+w^{\prime} \nu, e_{i_{0}}\right\rangle\right| \geq N$ for at least one $i_{0} \in I_{0}$. Furthermore, since $w, w^{\prime} \in W_{0}^{+}$we have $\left\langle w \nu+w^{\prime} \nu, e_{1}\right\rangle \geq 0$. The result now follows by taking $N \rightarrow \infty$, noting that $b<1$, and using the Riemann-Lebesgue Lemma.

Thus for all $\lambda \in P^{+}$we have

$$
h_{u}\left(A_{\lambda}\right)=P_{\lambda}(u)=\int_{\Omega}(u r)^{h(x, y ; \omega)} d \nu_{x}(\omega), \quad y \in V_{\lambda}(x) .
$$

As an application of (6.6) we compute the norms $\left\|A_{\lambda}\right\|, \lambda \in P^{+}$.

Theorem 6.3. Let $\lambda \in P^{+}$. Then $\left\|A_{\lambda}\right\|=P_{\lambda}(1)$.

Proof. Since $A \mapsto \hat{A}$ is an isometry, by Corollaries [5.5 and 5.8 we have

$$
\left\|A_{\lambda}\right\|=\left\|\hat{A}_{\lambda}\right\|_{\infty}=\sup \left\{\left|h\left(A_{\lambda}\right)\right|: h \in M_{2}\right\}=\sup \left\{\left|h_{u}\left(A_{\lambda}\right)\right|: u \in U\right\},
$$

where, as usual, $U=\mathbb{U}$ in the standard case and $U=\mathbb{U} \cup \mathbb{U}^{\prime}$ in the exceptional case. In the standard case this implies that $\left\|A_{\lambda}\right\|=P_{\lambda}(1)$, for by (6.6) we have $P_{\lambda}(1)>0$ and $\left|P_{\lambda}(u)\right| \leq P_{\lambda}(1)$ for all $u \in \mathbb{U}$ and $\lambda \in P^{+}$. In the exceptional case 
we only have $\left\|A_{\lambda}\right\| \geq P_{\lambda}(1)$, and so it remains to show that $\left\|A_{\lambda}\right\| \leq P_{\lambda}(1)$ in this case.

To see this, fix $o \in V_{P}$ and $\lambda \in P^{+}$. By Theorem 3.22 (iii), Corollary 3.23 and (6.6) we have $\left(A_{\lambda} F_{1}^{o}\right)(x)=P_{\lambda}(1) F_{1}^{o}(x)$ for all $x \in V_{P}$. Similarly, since $\lambda^{*}=\lambda$ here,

$$
\left(A_{\lambda}^{*} F_{1}^{o}\right)(x)=\left(A_{\lambda^{*}} F_{1}^{o}\right)(x)=\left(A_{\lambda} F_{1}^{o}\right)(x)=P_{\lambda}(1) F_{1}^{o}(x)
$$

for all $x \in V_{P}$. Since $F_{1}^{o}>0$ by (6.6), the Schur test (see [16 p.103] for example) implies that $\left\|A_{\lambda}\right\| \leq P_{\lambda}(1)$ (see also [8, Lemma 4.1]).

\section{Appendix A. Calculation of $q_{t_{\lambda}}$}

The formula in Proposition A.1 below is used in Proposition 3.15 to give an explicit formula for the Radon-Nikodym derivative in Theorem 3.17 It is also used after Proposition 2.1 to compare our formula (2.1) with the formula in 12 , Theorem 4.1.2] (in the case $P=Q$ ).

Proposition A.1. Let $\lambda \in P^{+}$. Then

$$
q_{t_{\lambda}}=\prod_{\alpha \in R^{+}} \tau_{\alpha}^{\langle\lambda, \alpha\rangle}
$$

Before proving the above proposition, we need some preliminary results.

Lemma A.2. Let $H$ be a wall of $\mathscr{X}$. Suppose that $\pi_{1}$ is a cotype $i$ (that is, cotype $\{i\})$ panel of $H$ and that $\pi_{2}$ is a cotype $j$ panel of $H$. Then $q_{i}=q_{j}$.

Proof. If $\sigma$ is any simplex of $\mathscr{X}$ and $c$ any chamber of $\mathscr{X}$, then there is a unique chamber, denoted $\operatorname{proj}_{\sigma}(c)$, nearest $c$ having $\sigma$ as a face [17, Corollary 3.9]. We show that the map $\varphi: \operatorname{st}\left(\pi_{1}\right) \rightarrow \operatorname{st}\left(\pi_{2}\right)$ given by $\varphi(c)=\operatorname{proj}_{\pi_{2}}(c)$ is a bijection (here $\operatorname{st}\left(\pi_{i}\right), i=1,2$, denotes the set of chambers of $\mathscr{X}$ having $\pi_{i}$ as a face). Observe first that if $c \in \operatorname{st}\left(\pi_{1}\right)$ then $\operatorname{proj}_{\pi_{1}}\left(\operatorname{proj}_{\pi_{2}}(c)\right)=c$. To see this, let $\mathcal{A}$ be any apartment containing $c$ and $H$ (see [17. Theorem 3.6]), and let $H^{+}$denote the half apartment of $\mathcal{A}$ containing $c$. Let $d$ be the unique chamber in $\operatorname{st}\left(\pi_{2}\right) \cap H^{+}$. It follows from 17 , Theorem 3.8] that $\operatorname{proj}_{\pi_{2}}(c)=d$, and so by symmetry $\operatorname{proj}_{\pi_{1}}(d)=c$. Similarly we have $\operatorname{proj}_{\pi_{2}}\left(\operatorname{proj}_{\pi_{1}}(d)\right)=d$ for all $d \in \operatorname{st}\left(\pi_{2}\right)$. So the map $\varphi$ is bijective.

Lemma A.2 allows us to make the following (temporary) definitions. Given a wall $H$ of $\mathscr{X}$, write $q_{H}=q_{i}$, where $i$ is the cotype of any panel of $H$. Now choose any apartment $\mathcal{A}$ of $\mathscr{X}$, and let $\psi: \mathcal{A} \rightarrow \Sigma$ be a type rotating isomorphism. For each $\alpha \in R$ and $k \in \mathbb{Z}$, write $q_{\alpha ; k}=q_{H}$, where $H=\psi^{-1}\left(H_{\alpha ; k}\right)$. We must show that this definition is independent of the particular $\mathcal{A}$ and $\psi$ chosen. To see this, let $\mathcal{A}^{\prime}$ be any (perhaps different) apartment of $\mathscr{X}$, and let $\psi^{\prime}: \mathcal{A}^{\prime} \rightarrow \Sigma$ be a type rotating isomorphism. Write $H^{\prime}=\psi^{\prime-1}\left(H_{\alpha ; k}\right)$. With $H$ as above, let $\pi$ be a panel of $H$, with cotype $i$, say, and so $q_{H}=q_{i}$. The isomorphism $\psi^{\prime-1} \circ \psi: \mathcal{A} \rightarrow \mathcal{A}^{\prime}$ is type rotating and sends $H$ to $H^{\prime}$. Thus $\left(\psi^{\prime-1} \circ \psi\right)(\pi)$ is a panel of $H^{\prime}$ with cotype $\sigma(i)$ for some $\sigma \in \operatorname{Aut}_{\mathrm{tr}}(D)$, and so $q_{H^{\prime}}=q_{\sigma(i)}=q_{i}=q_{H}$, by [15, Theorem 4.20].

Lemma A.3. Let $R$ be reduced. Then each wall of $\Sigma$ contains an element of $P$.

Proof. Each panel of each wall $H_{\alpha ; k}, \alpha \in R, k \in \mathbb{Z}$, contains $n-1$ vertices whose types are pairwise distinct. Since $R$ is reduced, the good vertices are simply the special vertices, that is, the elements of $P$ (see [2, VI, §2, No.2, Proposition 3]). Thus when there are two or more good types the result follows. 
This leaves the cases $E_{8}, F_{4}$ and $G_{2}$. Since $H_{-\alpha ; k}=H_{\alpha ;-k}$ it suffices to prove the result when $\alpha \in R^{+}$. Using the data in [2, Plates VII-IX] we see that for each $\alpha=\sum_{i=1}^{n} a_{i} \alpha_{i} \in R^{+}$there is an index $i_{1}$ such that $a_{i_{1}}=1$, or a pair of indices $\left(i_{2}, i_{3}\right)$ such that $a_{i_{2}}=2$ and $a_{i_{3}}=3$. In the former case $k \lambda_{i_{1}} \in H_{\alpha ; k}$, and in the latter case $\frac{k}{2} \lambda_{i_{2}} \in H_{\alpha ; k}$ if $k$ is even, and $\frac{k-3}{2} \lambda_{i_{2}}+\lambda_{i_{3}} \in H_{\alpha ; k}$ if $k$ is odd.

Proposition A.4. If $R$ is reduced, then $q_{\alpha ; k}=q_{\alpha}$ for all $\alpha \in R$ and $k \in \mathbb{Z}$.

Proof. The proof consists of the following steps:

(i) $q_{w \alpha ; 0}=q_{\alpha ; 0}$ for all $\alpha \in R$ and $w \in W_{0}$.

(ii) $q_{\alpha_{i} ; 0}=q_{\alpha_{i}}$ for each $i=1, \ldots, n$.

(iii) $q_{\alpha ; 0}=q_{\alpha}$ for all $\alpha \in R$.

(iv) $q_{\alpha ; k}=q_{\alpha ; 0}$ for all $\alpha \in R$ and $k \in \mathbb{Z}$.

(i) Let $\mathcal{A}$ be an apartment of $\mathscr{X}$, and let $\psi: \mathcal{A} \rightarrow \Sigma$ be a type rotating isomorphism. Write $H=\psi^{-1}\left(H_{\alpha ; 0}\right)$, so that $q_{\alpha ; 0}=q_{H}$. Let $w \in W_{0}$. Now the isomorphism $\psi^{\prime}=w \circ \psi: \mathcal{A} \rightarrow \Sigma$ is type rotating, and $\psi^{\prime-1}\left(H_{w \alpha ; 0}\right)=\psi^{-1}\left(H_{\alpha ; 0}\right)=H$. Thus $q_{w \alpha ; 0}=q_{H}=q_{\alpha ; 0}$.

(ii) Let $C_{0}$ be the fundamental chamber of $\Sigma$, and for each $i=1, \ldots, n$ let $C_{i}=s_{i} C_{0}$. Let $\mathcal{A}$ and $\psi: \mathcal{A} \rightarrow \Sigma$ be as in (i), and write $H=\psi^{-1}\left(H_{\alpha_{i} ; 0}\right)$. Then $\delta\left(\psi^{-1}\left(C_{0}\right), \psi^{-1}\left(C_{i}\right)\right)=s_{\sigma(i)}$ for some $\sigma \in \operatorname{Aut}_{\mathrm{tr}}(D)$, and so $q_{\alpha_{i} ; 0}=q_{H}=q_{\sigma(i)}$, and so by [15. Theorem 4.20] $q_{\alpha_{i} ; 0}=q_{i}=q_{\alpha_{i}}$.

(iii) Each $\alpha \in R$ is equal to $w \alpha_{i}$ for some $w \in W_{0}$ and some $i$, and so (iii) follows from (i) and (ii).

(iv) Let $\alpha \in R$ and $k \in \mathbb{Z}$. By Lemma A.3 there exists $\lambda \in H_{\alpha ; k} \cap P$, and so $H_{\alpha ; k}=t_{\lambda}\left(H_{\alpha ; 0}\right)$. Let $\mathcal{A}$ and $\psi$ be as in (i), and write $H=\psi^{-1}\left(H_{\alpha ; k}\right)$, so that $q_{\alpha ; k}=q_{H}$. The map $\psi^{\prime}=t_{\lambda}^{-1} \circ \psi: \mathcal{A} \rightarrow \Sigma$ is a type rotating isomorphism, and $\psi^{\prime-1}\left(H_{\alpha ; 0}\right)=\psi^{-1}\left(H_{\alpha ; k}\right)=H$. Thus $q_{\alpha ; k}=q_{H}=q_{\alpha ; 0}$.

We need an analogue of Proposition A.4 when $R$ is of type $B C_{n}$ for some $n \geq 1$. Observe that if $\alpha \in R_{1} \backslash R_{3}$, then $\alpha / 2 \in R_{2} \backslash R_{3}$, and $H_{\alpha ; 2 k}=H_{\alpha / 2 ; k}$ for all $k \in \mathbb{Z}$. Thus we define $\mathcal{H}_{1}=\left\{H_{\alpha ; k} \mid \alpha \in R_{1} \backslash R_{3}, k\right.$ odd $\}, \mathcal{H}_{2}=\left\{H_{\alpha ; k} \mid \alpha \in R_{2} \backslash R_{3}, k \in \mathbb{Z}\right\}$ and $\mathcal{H}_{3}=\left\{H_{\alpha ; k} \mid \alpha \in R_{3}\right\}$. Then $\mathcal{H}=\mathcal{H}_{1} \cup \mathcal{H}_{2} \cup \mathcal{H}_{3}$, where the union is disjoint. We have

$$
q_{\alpha ; k}= \begin{cases}q_{0} & \text { if } H_{\alpha ; k} \in \mathcal{H}_{1} \\ q_{n} & \text { if } H_{\alpha ; k} \in \mathcal{H}_{2} \\ q_{\alpha} & \text { if } H_{\alpha ; k} \in \mathcal{H}_{3}\end{cases}
$$

We omit the details of this calculation.

Remark A.5. Proposition A.4 and formula A.1 give the connection between our definitions of $R$ and $\tau_{\alpha}$ and Macdonald's definitions of $\Sigma_{1}$ and $q_{a}$ [12, §3.1].

Recall that for $\tilde{w} \in \tilde{W}$ we define $\mathcal{H}(\tilde{w})=\left\{H \in \mathcal{H} \mid H\right.$ separates $C_{0}$ and $\left.\tilde{w} C_{0}\right\}$. Also, observe that each $H \in \mathcal{H}$ is equal to $H_{\alpha ; k}$ for some $\alpha \in R_{1}^{+}$and some $k \in \mathbb{Z}$, and if $H_{\alpha ; k}=H_{\alpha^{\prime} ; k^{\prime}}$ with $\alpha, \alpha^{\prime} \in R_{1}^{+}$and $k, k^{\prime} \in \mathbb{Z}$, then $\alpha=\alpha^{\prime}$ and $k=k^{\prime}$.

Proof of Proposition A.1. Write $t_{\lambda}=t_{\lambda}^{\prime} g_{l}$, where $t_{\lambda}^{\prime} \in W$ and $l=\tau(\lambda)$. Then $\mathcal{H}\left(t_{\lambda}\right)=\mathcal{H}\left(t_{\lambda}^{\prime}\right)$ and $q_{t_{\lambda}}=q_{t_{\lambda}^{\prime}}$. Suppose that $t_{\lambda}^{\prime}=s_{i_{1}} \cdots s_{i_{m}}$ is a reduced expression 
for $t_{\lambda}^{\prime}$. Writing $H_{i}=H_{\alpha_{i} ; 0}$ if $i=1, \ldots, n$ and $H_{0}=H_{\tilde{\alpha} ; 1}$, we have

$$
\begin{aligned}
\mathcal{H}\left(t_{\lambda}\right) & =\left\{H_{i_{1}}, s_{i_{1}} H_{i_{2}}, \ldots, s_{i_{1}} \cdots s_{i_{m-1}} H_{i_{m}}\right\} \\
& =\left\{H_{\alpha ; k_{\alpha}} \mid \alpha \in R_{1}^{+} \text {and } 0<k_{\alpha} \leq\langle\lambda, \alpha\rangle\right\}
\end{aligned}
$$

where the hyperplanes in each set are pairwise distinct (see [11 Theorem 4.5]). If $1 \leq r \leq m$ and if $s_{i_{1}} \cdots s_{i_{r-1}} H_{i_{r}}=H_{\alpha ; k}$ then it is easy to see that $q_{\alpha ; k}=q_{i_{r}}$. Then using (A.2), Proposition A.4 and the fact that $\langle\lambda, \alpha\rangle \in 2 \mathbb{Z}$ for all $\alpha \in R_{1} \backslash R_{3}$, we have

$$
q_{t_{\lambda}}=\prod_{r=1}^{m} q_{i_{r}}=\prod_{\alpha \in R_{1}^{+}} \prod_{k_{\alpha}=1}^{\langle\lambda, \alpha\rangle} q_{\alpha ; k_{\alpha}}=\left[\prod_{\alpha \in R_{3}^{+}} q_{\alpha}^{\langle\lambda, \alpha\rangle}\right]\left[\prod_{\alpha \in R_{1}^{+} \backslash R_{3}^{+}}\left(q_{0} q_{n}\right)^{\langle\lambda, \alpha\rangle / 2}\right],
$$

and the result follows by direct calculation.

Proposition A.6 below is used to see that the formulae (1.8) and [15 (6.4)] are equivalent. It also gives the connection between our numbers $q_{w}, w \in W_{0}$, and Macdonald's numbers $t_{w}, w \in W_{0}$, from [13 (3.8)] (which is needed in the proof of Lemma B.2.

Recall that $R_{2}=\left\{\alpha \in R \mid \frac{1}{2} \alpha \notin R\right\}$. The inversion set of $w \in W_{0}$ is

$$
R_{2}(w)=\left\{\alpha \in R_{2}^{+} \mid H_{\alpha} \text { is between } C_{0} \text { and } w^{-1} C_{0}\right\},
$$

and we write $R(w)=\left\{\alpha \in R^{+} \mid H_{\alpha}\right.$ is between $C_{0}$ and $\left.w^{-1} C_{0}\right\}$.

Proposition A.6. For $w \in W_{0}$,

$$
q_{w}=\prod_{\alpha \in R_{2}\left(w^{-1}\right)} q_{\alpha}=\prod_{\alpha \in R\left(w^{-1}\right)} \tau_{\alpha}
$$

In particular,

$$
q_{w_{0}}=\prod_{\alpha \in R^{+}} \tau_{\alpha}
$$

(here $w_{0}$ is the longest element of $W_{0}$ ).

Proof. For $w \in W_{0}$ we have $R_{2}(w)=\left\{\alpha \in R_{2}^{+} \mid w \alpha \in R^{-}\right\}$, and if $w=s_{i_{1}} \cdots s_{i_{p}}$ is a reduced expression then

$$
R_{2}(w)=\left\{\alpha_{i_{p}}, s_{i_{p}} \alpha_{i_{p-1}}, \ldots, s_{i_{p}} \cdots s_{i_{2}} \alpha_{i_{1}}\right\}
$$

(see [2, VI, §1, No.6, Corollary 2 to Proposition 17]). The first equality in A.3 follows.

The second equality in A.3) is clear if $R$ is reduced (for $R_{2}=R$ and $\tau_{\alpha}=q_{\alpha}$ ). If $R$ is of type $B C_{n}$, and if $\alpha \in R_{2}\left(w^{-1}\right) \backslash R_{3}$, then $q_{\alpha}=q_{n}=\tau_{\alpha} \tau_{2 \alpha}$, verifying the result in this case too.

\section{Appendix B. Convex Hull}

Given a subset $X \subset V_{P}$, define the convex hull of $X$, or $\operatorname{conv}(X)$, to be the set of good vertices that lie in the intersection of all half-apartments that contain $X$.

Let $H_{\alpha ; k}$ be a hyperplane of $\Sigma$. The (closed) half-spaces of $\Sigma$ associated to $H_{\alpha ; k}$ are $H_{\alpha ; k}^{+}=\{z \in E \mid\langle z, \alpha\rangle \geq k\}$ and $H_{\alpha ; k}^{-}=\{z \in E \mid\langle z, \alpha\rangle \leq k\}$. This gives an analogous definition of $\operatorname{conv}(X)$ for subsets $X \subset P$, with the word half-apartment replaced by half-space.

Let $\leq$ denote the partial order on $P^{+}$given by $\mu \leq \lambda$ if and only if $\lambda-\mu \in P^{+}$. Note that this is quite different to the partial order $\preceq$ on $P$ used earlier. 
Lemma B.1. Let $\lambda \in P^{+}$. Then $\operatorname{conv}\{0, \lambda\}=\left\{\mu \in P^{+} \mid \mu \leq \lambda\right\}$.

Proof. Let $\mu \leq \lambda$ and write $\nu=\lambda-\mu \in P^{+}$. Suppose that $0, \lambda \in H_{\alpha ; k}^{ \pm}$. Since $H_{-\alpha ; k}=H_{\alpha ;-k}$ we may assume that $\alpha \in R^{+}$, and since $0 \in H_{\alpha ; k}^{ \pm}$the only cases to consider are $H_{\alpha ; k}^{-}$with $k \geq 0$, and $H_{\alpha ; k}^{+}$with $k \leq 0$. In the case $k \geq 0$ we have $\langle\mu, \alpha\rangle=\langle\lambda-\nu, \alpha\rangle \leq\langle\lambda, \alpha\rangle \leq k$ and so $\mu \in H_{\alpha ; k}^{-}$. In the case $k \leq 0$ we have $\langle\mu, \alpha\rangle \geq 0 \geq k$ and so $\mu \in H_{\alpha ; k}^{+}$. Thus $\left\{\mu \in P^{+} \mid \mu \leq \lambda\right\} \subseteq \operatorname{conv}\{0, \lambda\}$.

Now suppose that $\mu \in \operatorname{conv}\{0, \lambda\}$. Since $0, \lambda \in H_{\alpha_{i} ; 0}^{+}$for each $i \in I_{0}$, we have $\mu \in H_{\alpha_{i} ; 0}^{+}$for each $i \in I_{0}$ too, and so $\mu \in P^{+}$. Also, $0, \lambda \in H_{\alpha_{i} ;\left\langle\lambda, \alpha_{i}\right\rangle}^{-}$for each $i \in I_{0}$, and so $\left\langle\mu, \alpha_{i}\right\rangle \leq\left\langle\lambda, \alpha_{i}\right\rangle$ for each $i \in I_{0}$. That is, $\lambda-\mu \in P^{+}$, and so $\mu \leq \lambda$.

Lemma B.2. Let $\lambda, \mu \in P^{+}$. Then $\left|V_{\lambda}(x) \cap V_{\mu^{*}}(y)\right|=1$ whenever $y \in V_{\lambda+\mu}(x)$.

Proof. We give a proof using calculations involving the Macdonald spherical functions. We note that it is also possible to give a purely 'building theoretic' proof.

For $\lambda \in P^{+}$let

$$
P_{\lambda}^{\prime}(x)=\frac{W_{0}\left(q^{-1}\right)}{W_{0 \lambda}\left(q^{-1}\right)} q_{t_{\lambda}}^{1 / 2} P_{\lambda}(x)=q_{t_{\lambda}}^{-1 / 2} N_{\lambda} P_{\lambda}(x),
$$

where we have used Proposition 1.5 and define the monomial symmetric function $m_{\lambda}(x) \in \mathbb{C}[P]^{W_{0}}$ by

$$
m_{\lambda}(x)=\sum_{\mu \in W_{0} \lambda} x^{\mu}
$$

The set $\left\{m_{\lambda}(x)\right\}_{\lambda \in P^{+}}$forms a basis for $\mathbb{C}[P]^{W_{0}}$, and by the calculations made in [13, §10] (noting also Proposition A.6) we have

$$
P_{\lambda}^{\prime}(x)=\sum_{\mu \preceq \lambda} c_{\lambda, \mu} m_{\mu}(x), \text { where } c_{\lambda, \lambda}=1 .
$$

This is the so called triangularity condition of the Macdonald spherical functions.

It is clear that $m_{\lambda}(x) m_{\mu}(x)=\sum_{\nu \preceq \lambda+\mu} d_{\lambda, \mu ; \nu} m_{\nu}(x)$ where $d_{\lambda, \mu ; \lambda+\mu}=1$, and so it follows that

$$
P_{\lambda}^{\prime}(x) P_{\mu}^{\prime}(x)=\sum_{\nu \preceq \lambda+\mu} e_{\lambda, \mu ; \nu} P_{\nu}^{\prime}(x)
$$

for some numbers $e_{\lambda, \mu ; \nu}$ with $e_{\lambda, \mu ; \lambda+\mu}=1$. It follows that for all $\lambda, \mu, \nu \in P^{+}$,

$$
a_{\lambda, \mu ; \nu}=q_{t_{\lambda}}^{1 / 2} q_{t_{\mu}}^{1 / 2} q_{t_{\nu}}^{-1 / 2} \frac{N_{\nu}}{N_{\lambda} N_{\mu}} e_{\lambda, \mu ; \nu},
$$

and since $q_{t_{\lambda+\mu}}=q_{t_{\lambda}} q_{t_{\mu}}$ we have $a_{\lambda, \mu ; \lambda+\mu}=N_{\lambda+\mu} N_{\lambda}^{-1} N_{\mu}^{-1}$. It follows from (1.7) that $\left|V_{\lambda}(x) \cap V_{\mu^{*}}(y)\right|=1$ whenever $y \in V_{\lambda+\mu}(x)$, proving the result.

This immediately gives the following.

Corollary B.3. Let $\lambda \in P^{+}$and $\mu \leq \lambda$, and let $x, y \in V_{P}$ be any vertices with $y \in V_{\lambda}(x)$. There exists a unique vertex, denoted $v_{\mu}(x, y)$, in the set $V_{\mu}(x) \cap V_{\nu^{*}}(y)$, where $\nu=\lambda-\mu \in P^{+}$.

Theorem B.4. Let $\lambda \in P^{+}, x \in V_{P}$, and $y \in V_{\lambda}(x)$. Then

$$
\operatorname{conv}\{x, y\}=\left\{v_{\mu}(x, y) \mid \mu \leq \lambda\right\} .
$$


Proof. Let $H$ be a half-apartment of $\mathscr{X}$ containing $\{x, y\}$, and let $\mathcal{A}$ be any apartment containing $H$. It is easy to see (using Axiom (B2) of [4, p.76]) that there exists a type rotating isomorphism $\psi: \mathcal{A} \rightarrow \Sigma$ such that $\psi(x)=0$ and $\psi(y)=\lambda$. Let $\mu \leq \lambda$ and write $\nu=\lambda-\mu \in P^{+}$. The vertex $v=\psi^{-1}(\mu)$ is in both $V_{\mu}(x)$ and $V_{\nu^{*}}(y)$ (as $y \in V_{\nu}(v)$, for $\left(t_{-\mu} \circ \psi\right)(v)=0$ and $\left.\left(t_{-\mu} \circ \psi\right)(y)=\nu \in P^{+}\right)$, and so by Corollary B.3 $v_{\mu}(x, y)=v \in \mathcal{A}$. Now $\psi(H)$ is a half-space of $\Sigma$ which contains 0 and $\lambda$, and so by Lemma B.1 $\mu \in \psi(H)$. Thus $v_{\mu}(x, y)=\psi^{-1}(\mu) \in H$, showing that

$$
\left\{v_{\mu}(x, y) \mid \mu \leq \lambda\right\} \subseteq \operatorname{conv}\{x, y\} .
$$

Suppose now that $v \in \operatorname{conv}\{x, y\}$. Thus there exists an apartment $\mathcal{A}$ containing $x, y$ and $v$. Let $\psi: \mathcal{A} \rightarrow \Sigma$ be a type rotating isomorphism such that $\psi(x)=0$ and $\psi(y)=\lambda$, and write $\mu=\psi(v)$. If $\mu \notin \operatorname{conv}\{0, \lambda\}$ then there is a (closed) half-space of $\Sigma$ which contains 0 and $\lambda$ but not $\mu$, and it follows that there exists a half-apartment of $\mathcal{A}$ which contains $x$ and $y$ but not $v$, a contradiction. Thus $\mu \in \operatorname{conv}\{0, \lambda\}$, and so by Lemma B.1 $\mu \leq \lambda$. It follows that $v \in V_{\mu}(x) \cap V_{\nu^{*}}(y)$, where $\nu=\lambda-\mu \in P^{+}$, and so $v=v_{\mu}(x, y)$, completing the proof.

Note that the above shows that $\operatorname{conv}\{x, y\}$ is a finite set for all $x, y \in V_{P}$. Indeed we have the following corollary, which also shows that $|\operatorname{conv}\{x, y\}|=|\operatorname{conv}\{u, v\}|$ whenever $y \in V_{\lambda}(x)$ and $v \in V_{\lambda}(u)$, and that $|\operatorname{conv}\{x, y\}|$ does not depend on the parameters of the building.

Corollary B.5. Let $y \in V_{\lambda}(x)$. Then $|\{\operatorname{conv}\{x, y\}\}|=\prod_{i=1}^{n}\left(\left\langle\lambda, \alpha_{i}\right\rangle+1\right)$.

Proof. By Theorem B.4 we have $|\{\operatorname{conv}\{x, y\}\}|=\left|\left\{\mu \in P^{+} \mid \mu \leq \lambda\right\}\right|$. Now $\mu \leq \lambda$ if and only if $0 \leq\left\langle\mu, \alpha_{i}\right\rangle \leq\left\langle\lambda, \alpha_{i}\right\rangle$ for all $i=1, \ldots, n$. Thus

$$
|\{\operatorname{conv}\{x, y\}\}|=\mid\left\{\left(k_{1}, \ldots, k_{n}\right) \in \mathbb{N}^{n} \mid 0 \leq k_{i} \leq\left\langle\lambda, \alpha_{i}\right\rangle \text { for all } i=1, \ldots, n\right\} \mid,
$$

and the result follows.

We conclude this appendix with a sketch of the following theorem, which was used in the construction of the topology on $\Omega$. Recall the definition of the maps $\varphi_{\mu, \lambda}$ made in the paragraphs after the proof of Theorem 3.6

Theorem B.6. Fix $x \in V_{P}$ and define $\theta: \Omega \rightarrow \prod_{\lambda \in P^{+}} V_{\lambda}(x)$ by $\omega \mapsto\left(v_{\lambda}^{x}(\omega)\right)_{\lambda \in P^{+}}$. Then $\theta$ is a bijection of $\Omega$ onto $\lim _{(}\left(V_{\lambda}(x), \varphi_{\mu, \lambda}\right)$.

Proof. It is not too difficult to see that if $\mathcal{S}$ and $\mathcal{S}^{\prime}$ are sectors with the same good vertices, then $\mathcal{S}=\mathcal{S}^{\prime}$. Thus it is clear that $\theta$ is injective. To show that $\theta$ is surjective, let $\left(v_{\nu}\right)_{\nu \in P^{+}} \in \lim \left(V_{\lambda}(x), \varphi_{\mu, \lambda}\right)$. For each $m \geq 1$ let $\mu_{m}=m\left(\lambda_{1}+\cdots+\lambda_{n}\right)$, and let $\mathcal{C}(x ; m)$ denote the set of chambers contained in the intersection of all halfapartments containing $x$ and $v_{\mu_{m}}$. Since $\mu_{m} \in P^{++}$the sets $\mathcal{C}(x ; m)$ are nonempty for all $m \geq 1$, and $\mathcal{C}(x ; m) \subset \mathcal{C}(x ; k)$ whenever $m \leq k$. Furthermore, for $m \geq 1$ write $\mathcal{C}_{m}$ for the set of chambers of $\Sigma$ contained in the intersection of all half-spaces containing 0 and $\mu_{m}$.

For each $m \geq 1$ there exists an apartment $\mathcal{A}_{m}$ containing $x$ and $v_{\mu_{m}}$, and a type rotating isomorphism $\psi_{m}: \mathcal{A}_{m} \rightarrow \Sigma$ such that $\psi_{m}(x)=0$ and $\psi_{m}\left(v_{\mu_{m}}\right)=\mu_{m}$. Furthermore, if $\mathcal{A}_{m}^{\prime}$ and $\psi_{m}^{\prime}$ also have these properties, then it is easy to see that $\left.\psi_{m}\right|_{\mathcal{C}(x ; m)}=\left.\psi_{m}^{\prime}\right|_{\mathcal{C}(x ; m)}$. Also, $\left.\psi_{m+1}\right|_{\mathcal{C}(x ; m)}=\left.\psi_{m}\right|_{\mathcal{C}(x ; m)}$ for all $m \geq 1$.

For each $m \geq 1$ define $\xi_{m}: \mathcal{C}_{m} \rightarrow \mathscr{X}$ by $\xi_{m}=\left.\psi_{m}^{-1}\right|_{\mathcal{C}_{m}}$. Since $\left.\xi_{m+1}\right|_{\mathcal{C}_{m}}=\xi_{m}$ we have $\left.\xi_{k}\right|_{\mathcal{C}_{m}}=\xi_{m}$ for all $k \geq m$. We therefore define $\xi: \mathcal{C}\left(\mathcal{S}_{0}\right) \rightarrow \mathscr{X}$ by 
$\xi(C)=\xi_{m}(C)$ once $C \in \mathcal{C}_{m}$. By replacing the type map $\tau: V(\Sigma) \rightarrow I$ on $\Sigma$ by $\sigma_{i} \circ \tau$ where $i=\tau(x)$, we may take all of the above isomorphisms to be type preserving, and so by [17. Theorem 3.6] we see that $\xi$ extends to an isometry $\tilde{\xi}: \mathcal{C}(\Sigma) \rightarrow \mathscr{X}$. Then $\tilde{\xi}(\mathcal{C}(\Sigma))$ is an apartment of $\mathscr{X}$, and $\mathcal{S}=\tilde{\xi}\left(\mathcal{C}\left(\mathcal{S}_{0}\right)\right)$ is a sector. Let $\omega$ be the class of $\mathcal{S}$. Then $\theta(\omega)=\left(v_{\nu}\right)_{\nu \in P^{+}}$.

\section{REFERENCES}

[1] M.F. Atiyah and I. G. Macdonald. Introduction to Commutative Algebra. Addison-Wesley Series in Mathematics. Addison-Wesley, 1969.

[2] N. Bourbaki. Lie Groups and Lie Algebras, Chapters 4-6. Elements of Mathematics. SpringerVerlag, Berlin Heidelberg New York, 2002.

[3] N. Bourbaki. Theory of Sets. Elements of Mathematics. Springer-Verlag, Berlin Heidelberg New York, 2004.

[4] Kenneth Brown. Buildings. Springer-Verlag, New York, 1989.

[5] D. I. Cartwright. A Brief Introduction to Buildings, in Harmonic Functions on Trees and Buildings. Contemp. Math. Amer. Math. Soc., Providence, Rhode Island, 1997.

[6] D. I. Cartwright. Harmonic Functions on Buildings of type $\tilde{A}_{n}$. In: Picardello, M., Woess, W., (eds) Proceedings of the Conference 'Random Walks and Discrete Potential Theory', Cortona, Italy, June 22-28, Symposia Math, 38:104-138, 1997.

[7] D. I. Cartwright. Spherical Harmonic Analysis on Buildings of Type $\widetilde{A}_{n}$. Monatsh. Math., 133(2):93-109, 2001.

[8] D. I. Cartwright and W. Młotkowski. Harmonic Analysis for Groups Acting on Triangle Buildings. J. Austral. Math. Soc. (Series A), 56:345-383, 1994.

[9] Kenneth R. Davidson. $C^{*}$-Algebras by Example. Fields Institute Monographs. American Mathematical Society, Providence, Rhode Island, U.S.A., 1996.

[10] J. E. Humphreys. Introduction to Lie Algebras and Representation Theory, volume 9 of Graduate Texts in Mathematics. Springer-Verlag, New York-Berlin, 1978.

[11] J. E. Humphreys. Reflection Groups and Coxeter Groups, volume 29 of Cambridge Studies in Advanced Mathematics. C.U.P, Cambridge, 1990.

[12] I. G. Macdonald. Spherical Functions on a Group of p-adic type, volume No.2 of Publications of the Ramanujan Institute. Ramanujan Institute, Centre for Advanced Study in Mathematics, University of Madras, Madras, 1971.

[13] I. G. Macdonald. Orthogonal Polynomials Associated with Root Systems. Séminaire Lotharingien de Combinatoire, 45(Article B45a), 2000.

[14] I. G. Macdonald. Affine Hecke Algebras and Orthogonal Polynomials, volume 157 of Cambridge Tracts in Mathematics. C.U.P., Cambridge, 2003.

[15] J. Parkinson. Buildings and Hecke Algebras. Journal of Algebra, 297(1):1-49, 2006.

[16] Gert Pedersen. Analysis Now. Graduate Texts in Mathematics. Springer-Verlag, 1989.

[17] Mark Ronan. Lectures on Buildings. Perspectives in Mathematics. Academic Press, 1989. 